\title{
Regulatory mechanisms, functions, and clinical significance of CircRNAs in triple-negative breast cancer
}

\author{
Lijuan Lyu ${ }^{1,2 \dagger}$, Shizhen Zhang ${ }^{3 \dagger}$, Yujiao Deng ${ }^{1,2}$, Meng Wang ${ }^{2}$, Xinyue Deng ${ }^{1}$, Si Yang ${ }^{1,2}$, Ying Wu $\mathbf{u}^{1,2}$ \\ and Zhijun Dai ${ }^{1,2^{*}}$
}

\begin{abstract}
Circular RNAs (circRNAs) are a new class of endogenous regulatory RNAs characterized by covalently closed cyclic structure lacking poly-adenylated tails, and are capable of regulating gene expression at transcription or post-transcription levels. Recently, plentiful circRNAs have been discovered in breast cancer and some circRNAs expression profiles are specifically involved in the triple-negative breast cancer (TNBC). TNBC is a type of malignant tumor defined by the lack of estrogen receptor, progesterone receptor, and human epidermal growth factor receptor 2 expression. Considering its clinical characteristics of high invasion, metastasis, poor prognosis, and lack of effective response to conventional chemotherapies or targeted therapies, it could be a promosing option to discover specific circRNAs as new targets for TNBC treatment. Meanwhile, accumulating evidence has demonstrated that circRNAs are dysregulated in TNBC tissues and are correlated with clinicopathological features and prognosis of TNBC patients. Furthermore, looking for circRNAs with high specificity and sensitivity will provide a new opportunity for the early diagnosis, clinical treatment, and prognosis monitoring of TNBC. Herein, we reviewed the biogenesis, regulatory mechanisms, and biological functions of circRNAs in TNBC and summarized the relationship between circRNAs expression and the clinicopathology, diagnosis, and prognosis of patients with TNBC.
\end{abstract}

Keywords: CircRNAs, Triple-negative breast cancer, Biogenesis, Biological functions, Regulation mechanisms, Diagnosis, Clinicopathology, Prognosis

\section{Background}

Breast cancer $(\mathrm{BC})$ is the most common malignant disease among females and seriously threatens the health of women worldwide [1, 2]. Triple-negative breast cancer $(\mathrm{TNBC})$ is the subtype of $\mathrm{BC}$ with the highest recurrence, metastasis, and mortality rate. It is pathologically characterized by the absence of estrogen receptor (ER), progesterone receptor (PR), and human epidermal growth factor receptor 2 (HER2) [3, 4]. TNBC accounts

\footnotetext{
*Correspondence: dzj0911@126.com

'Lijuan Lyu and Shizhen Zhang contributed equally to this work.

1 Department of Breast Surgery, The First Affiliated Hospital, College of Medicine, Zhejiang University, Hangzhou 310003, China

Full list of author information is available at the end of the article
}

for approximately $15 \%$ of all BCs, with typically more aggressive characteristics and lack of effective targeted treatment options $[5,6]$. Therefore, early detection and feasible targeted therapy are especially important for TNBC patients. Traditionally, many clinicopathological features, such as tumor size, lymph node status, and histological grade, are associated with patient outcomes and are used to predict patient prognosis [7]. Several newly identified biomarkers, such as tumor-associated macrophages (TAMs), microRNAs (miRNAs), and long non-coding RNA (lncRNAs), also have important prognostic values [8]. In recent years, circular RNAs (circRNAs) have attracted a lot of attention due to their key roles involved in human cancers, including TNBC. 
CircRNA was first observed in the 1976 by Sanger et al. in plant-infected viroids by electron microscopy and were considered pathogenic because of their structural similarity to viruses [9]. They were later discovered in eukaryotes and were thought to be a result of splicing errors for several decades after the 1970s [10,11]. However, more recent studies of circRNAs in drosophila, mouse, and other eukaryotes indicate that these RNA transcripts are evolutionarily conserved and thus are not simple artifacts of faulty splicing $[12,13]$. In addition, with the advances in sequencing technology and bioinformatics analyses, the abundance and diversity of circRNAs therefore can been easily identified [14, 15]. High-throughput RNA sequencing (RNA-seq) and microarray are widely used technology to annotate new RNA species and quantify RNA abundance, which have identified the majority of circRNAs in human cells. Besides, increasing bioinformatic algorithms have been developed for identifying circRNAs, such as circRNA_finder, find_circ, CIRCexplorer, CIRI, and MapSplice [16]. The mainly validation methods for circRNA expression are quantitative real-time PCR (qRT-PCR) and Northern blotting, and Northern blotting is a more stringent circRNA validation method than qRT-PCR, given its straightforward procedure with no reverse transcription and amplification steps [17].

Researchers have identified that circRNAs possess significant roles in regulation of multiple factors at transcription or post-transcriptional levels in mammalian cells, and dysregulations of circRNAs can affect genes expression and lead to diseases [18-20], including cancer $[21,22]$. Many studies using microarray and RNA-seq revealed that circRNAs can be frequently detected in BC $[23,24]$. Especially, recent studies depicted the systematic profiling and characterization of circRNA expression pattern in different subtypes of BC $[25,26]$, and such subtype-specific set of circRNAs may be used for distinguishing the tumor subtypes, suggesting that circRNAs can be exploited as novel molecular biomarkers. Notably, more and more evidence have indicated that dysregulation of circRNAs participate in carcinogenesis and progression of TNBC, as a result, certain circRNAs could be potentially diagnostic and prognostic biomarkers or therapeutic targets for TNBC [27-29]. Hence, we concentrated on recent findings related to the role of circRNAs in TNBC and summarized their potential clinical implications in TNBC, such as identification of biomarkers for early and differential diagnosis, prognosis, and prediction of response to specific therapies.

\section{The biogenesis of circRNAs}

CircRNAs are derived from precursor messenger RNAs (pre-mRNAs), which are transcribed by RNA polymerase II, and characterized by circular shapes resulting from covalently closed continuous loops [30,31]. With their unique structures, circRNAs are resistant to exonuclease RNase Rand, which makes them more conservative and stable than their linear counterparts [32]. CircRNAs are mainly divided into four types according to their various components and circularization mechanism, including exon circRNAs (EcircRNAs), circular intronic RNAs (ciRNAs), exon-intron circRNAs (EIciRNAs), and intergenic circRNAs or fusion circRNAs (f-circRNAs) [33]. EcircRNAs, consisting of only one exon or multiple quantities of exons and forming through a shearing process called "head-to-tail" or "backsplicing", make up over $80 \%$ of circRNAs and mostly exist in the cytoplasm [34]. EIciRNAs are predominantly located in the nucleus and is circularized in the form of retaining introns between exons [35]. There are currently three models, namely intron-pairing-driven circularization, RNA-binding-protein (RBP)-dependent circularization, and lariat-driven circularization, that have been recognized to elaborate the origination of EcircRNAs and EIciRNAs (Fig. 1) [36]. Notably, a newly discovered type of circRNA termed ciRNAs, are derived from introns and mainly found in the nucleus. There are also three hypothetical models explaining the formation of ciRNAs, including circular RNA from group I introns, circular RNA from group II introns, and intron RNA lariat (Fig. 2) [36, 37]. The f-circRNAs are identifed by applying CIRI (an algorithm for de novo circular RNA identification) and contain two intronic circRNA fragments flanked by GT-AC splicing signals acting as the splice donor and acceptor of the circular junction while forming an integrated circRNA (Fig. 1d) [38].

\section{Regulatory mechanisms of circRNAs in TNBC}

CircRNAs are a novel class of abundant, stable and ubiquitous noncoding RNAs with diverse regulatory roles in tumor cells, including serving as miRNA sponges, binding to RBPs, modulating genes transcription, competing with linear splicing, translating into protein, and so on [20,39]. In TNBC, the circRNAs has not yet been found to modulate genes transcription and compete with linear splicing. The regulatory mechanisms of circRNAs involved in TNBC cells are depicted in Fig. 3 and Table 1, which summarized most circRNAs involved in TNBC.

\section{CircRNAs serve as miRNA sponges}

MiRNAs negatively regulate the gene expression of messenger RNAs (mRNAs) through direct base pairing to target sites in mRNA $3^{\prime}$ untranslated regions, eventually leading to decreased mRNA stability and translation suppression [40]. The competing endogenous RNA (ceRNA) hypothesis showed that other RNAs with miRNA target sites can compete with mRNAs for miRNA binding [41]. 

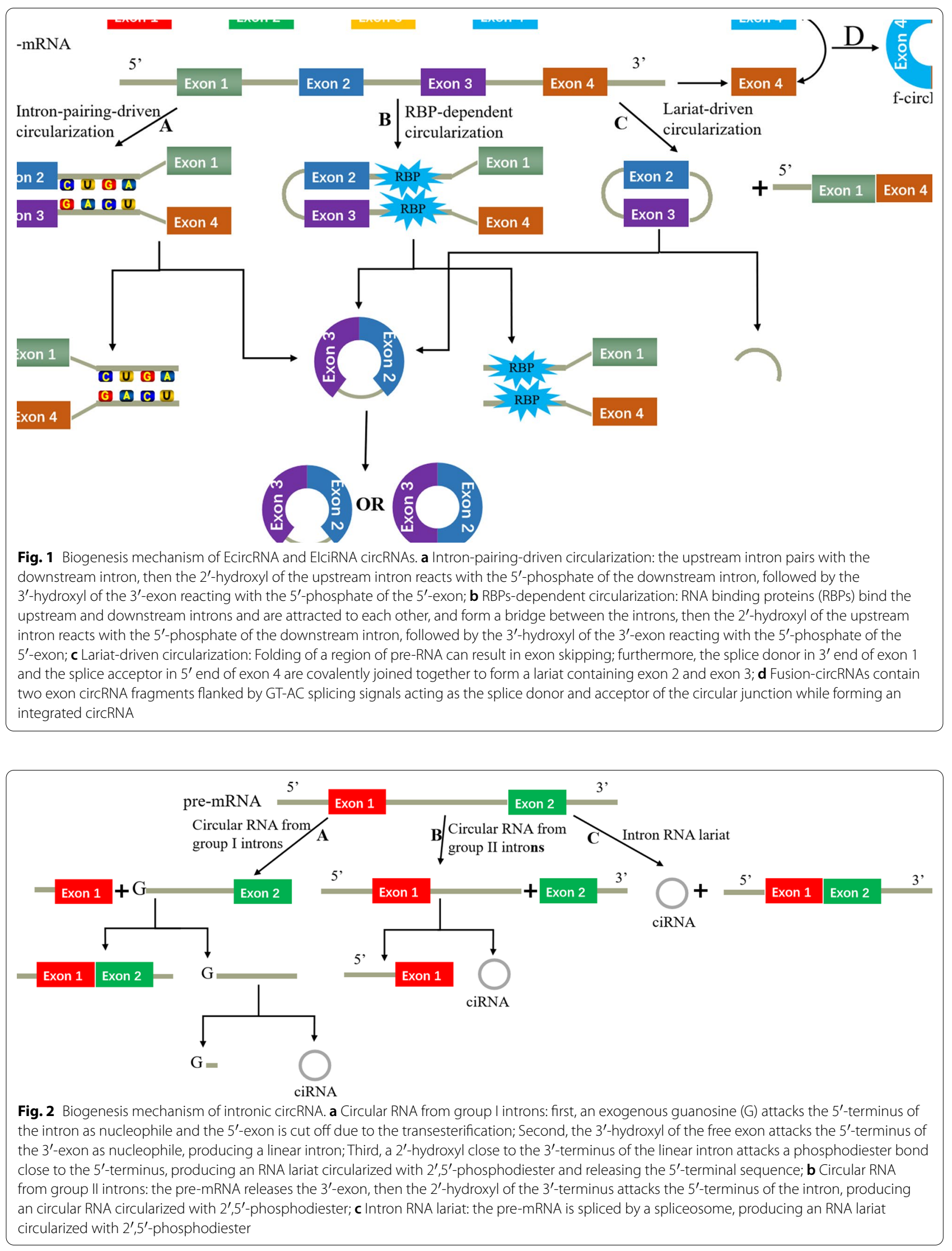


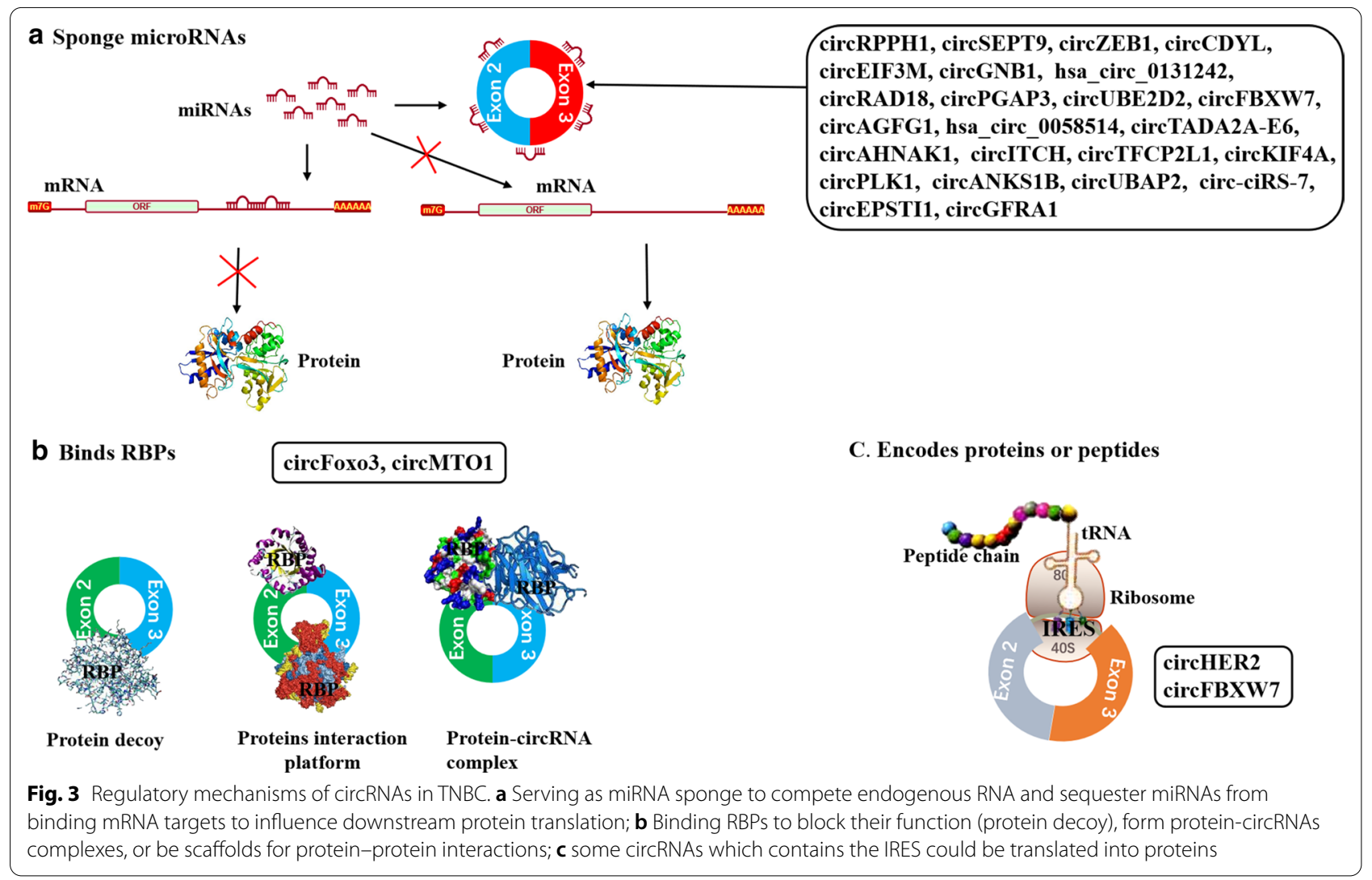

Indeed, most circRNAs, containing a large number of different types of miRNA response elements, are located in the cytoplasm with huge miRNA-binding capacity and have been found to interact with miRNA and serve as miRNA sponges to remove the inhibitory effect of miRNA on its target genes in cancer [42-44].

Most of the circRNAs currently reported in TNBC serve as miRNA sponges (Fig. 3a). For instance, ciRS7 , an earlier discovered circRNA in TNBC, contains 20 miR-1299-binding sites and functions as a ceRNA of miR-1299 to enhance the expression of the matrix metalloproteinase family members, thereby contributing to the high migration and invasion properties of TNBC cells [45]. CircEPSTI1 promotes TNBC proliferation and apoptosis by upregulating $B C L 11 A$ expression via binding to $m i R-4753$ and $m i R-6809$ [46]. Zeng et al. [47] reported that $\operatorname{circANKS1B}$ abundantly sponged miR$148 a-3 p$ and $m i R-152-3 p$ to increase the expression of transcription factor USF1. Moreover, the splicing factor ESRP1, regulated by USF1, can promote circANKS1B biogenesis in TNBC. CircTADA2A-E6 preferentially acts as an $m i R-203 a-3 p$ sponge to restore the expression of miRNA target gene SOCS3, resulting in a less aggressive oncogenic phenotype [48]. Zheng et al. demonstrated that $\operatorname{circSEPT} 9$ could regulate the expression of $L I F$ via sponging $m i R-637$ and activating the LIF/ Stat3 signaling pathway involved in the progression of TNBC. More importantly, they discovered that E2F1 and EIF4A3 enhance the expression of circSEPT9 by binding to the SEPT9 promoter and pre-mRNA [49]. CircGFRA1 was found not only could upregulate TLR4 via sponging for miR-361-5p, thus affecting the sensitivity of TNBC cells to paclitaxel (PTX) [50], but also upregulate its parent gene GFRA1 expression through sponging $m i R-34 a$, thus promoting proliferation and inhibiting apoptosis of TNBC cells [29]. Likewise, circKIF4A and circITCH were proved to upregulate its parental genes via acting as sponges for miRNAs thereby mediating TNBC progress [51, 52]. Additionally, more miRNA-sponge functions of circRNAs have been and are being validated in TNBC, including circUBAP2/miR-661/MTA1 [53], circPLK1/miR296-5p/PLK1 [54], circTFCP2L1/miR-7/PAK1 [55], circAHNAK1/miR-421/RASA1 [56], circAGFG1/miR195-5p/CCNE1 [57], circZEB1/miR-448/eEF2K [58], circGNB1/miR-141-5p/IGF1R [59], circRPPH1/miR556-5p/YAP1 [60], circCDYL/miR-190a-3p/TP53INP1 [61], circEIF3M/miR-33a/cyclinD1 [62], circUBE2D2/ miR-512-3p/CDCA3 [63], and $\operatorname{circPGAP3/miR-330-3p/~}$ $M y c$ [64]. 


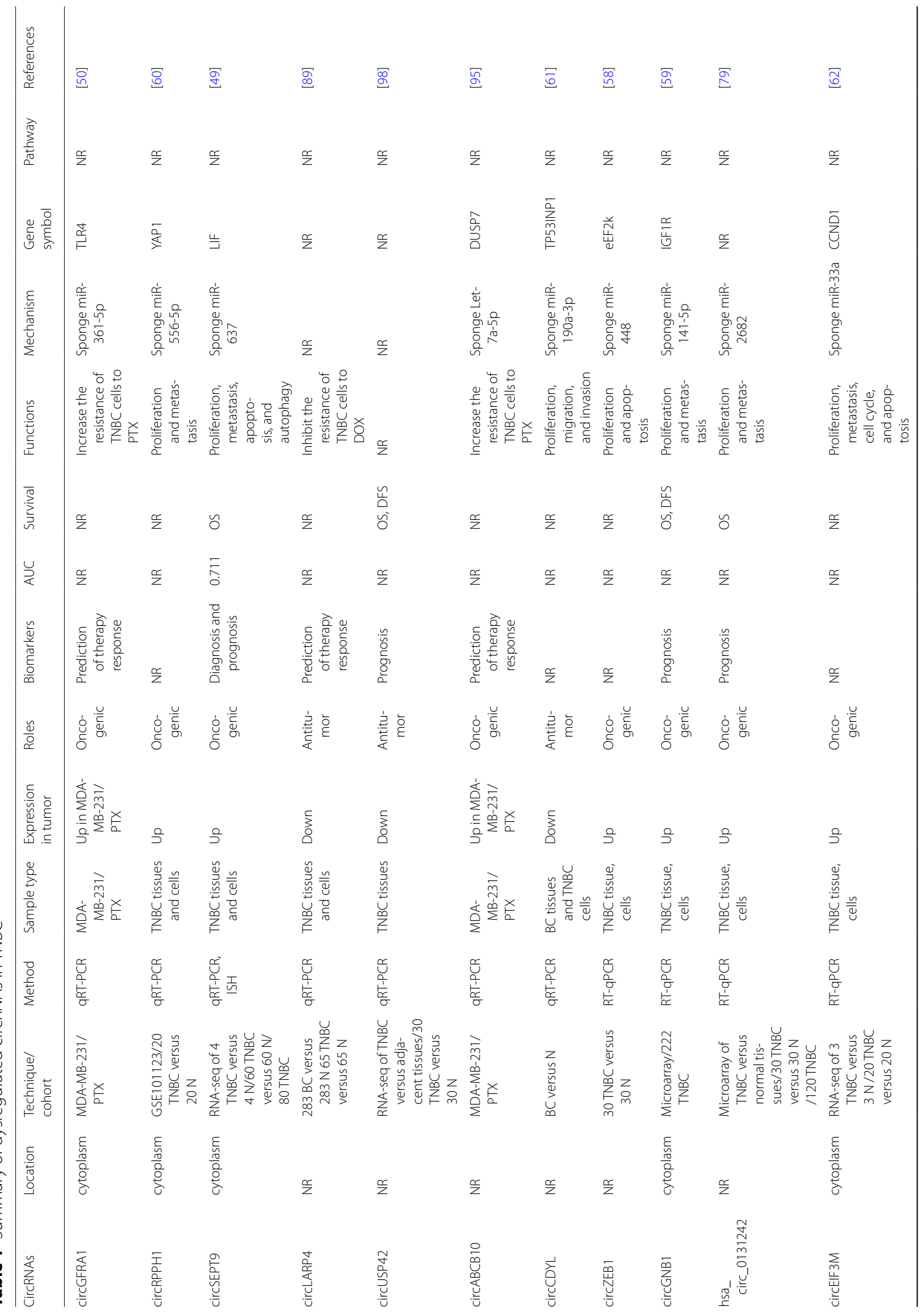




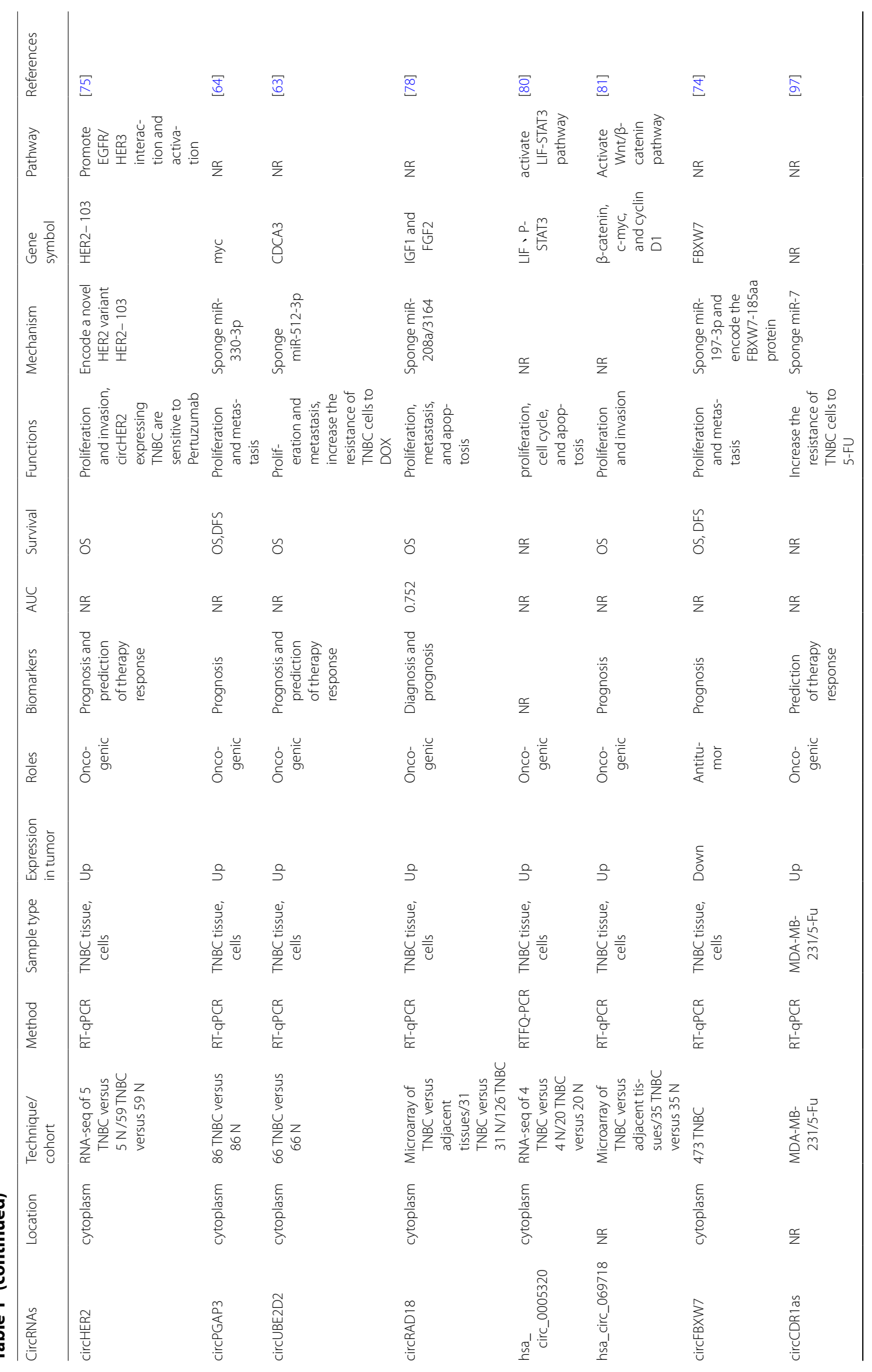


Lyu et al. J Hematol Oncol (2021) 14:41

Page 7 of 18

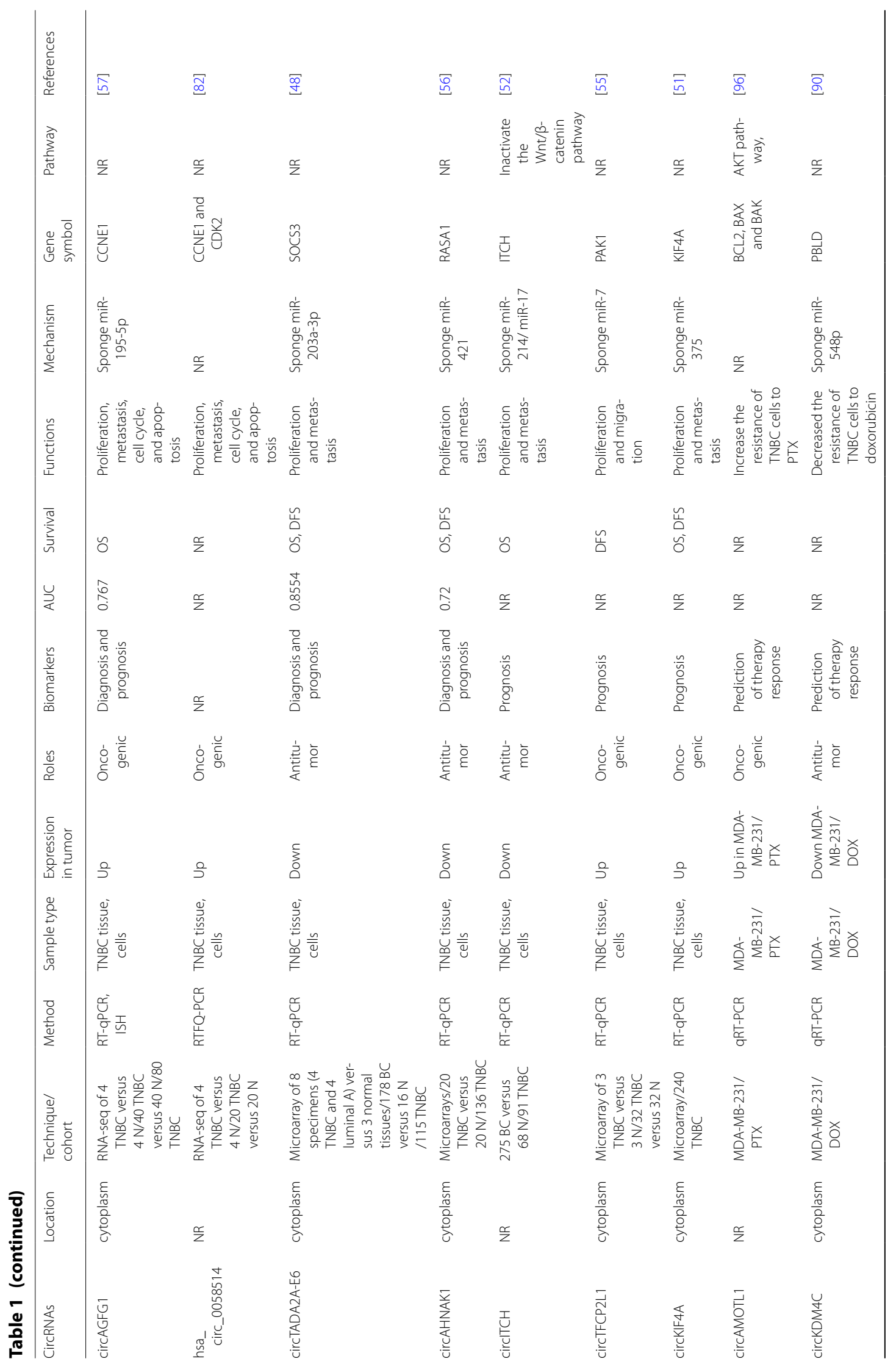




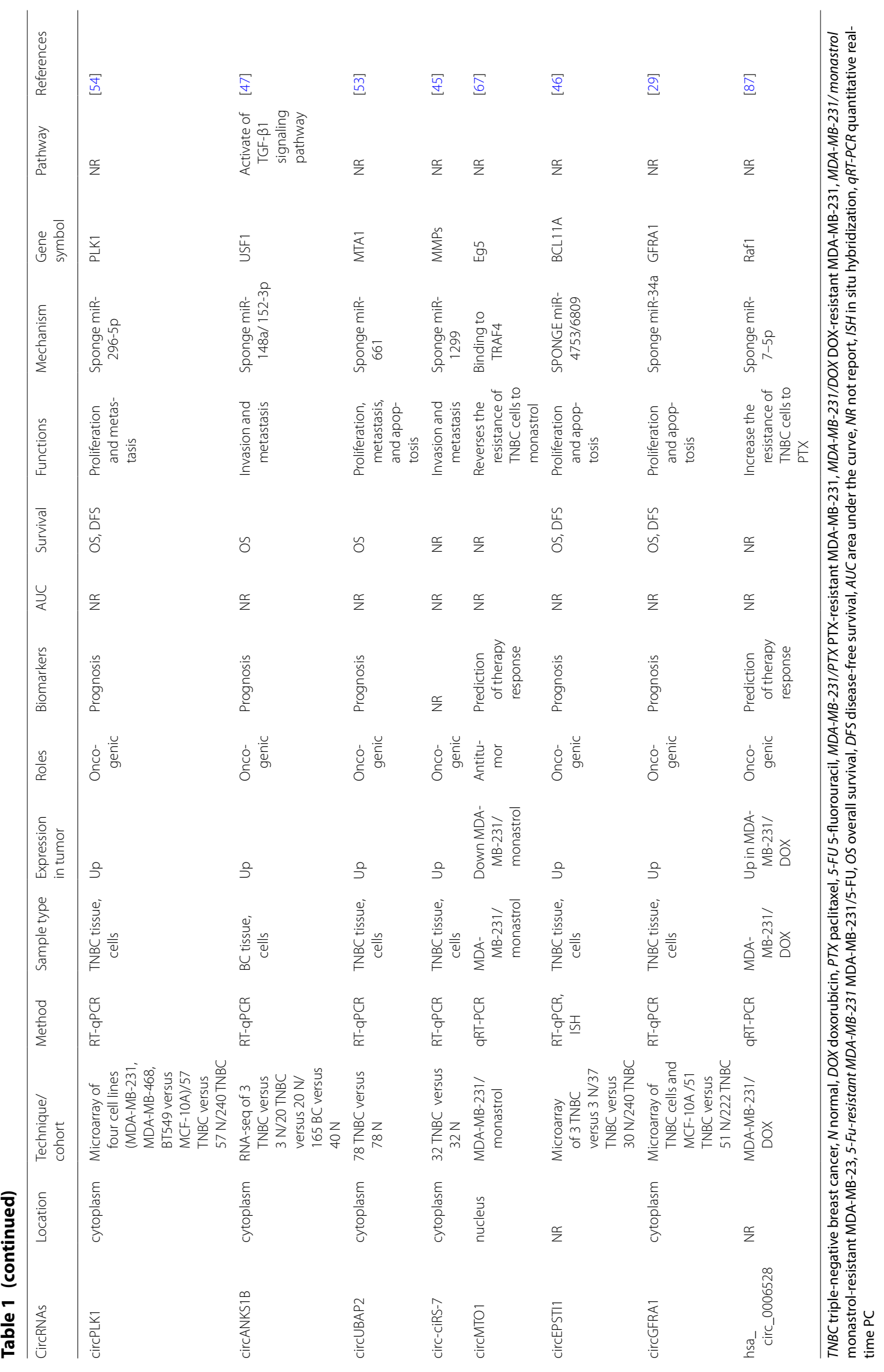




\section{CircRNAs interact with RBPs}

CircRNAs could specifically bind to proteins directly or through RNA as well as sequester proteins to block the protein effects by working as competing elements (Fig. 3b) [65]. One classic example of circRNA to interact with proteins is circFoxo3. It's expression significantly promoted TNBC cell apoptosis with upregulation of Foxo3, but downregulation of p53. Mechanically, circFoxo 3 prefered to bind MDM2 and p53, instead of Foxo3 in MDA-MB-231 cells. As such, circFoxo3 overexpression promoted MDM2-induced p53 ubiquitination and subsequent degradation, but competitively prevented MDM2-mediated Foxo3 ubiquitination and degradation, eventually leading to cell apoptosis due to upregulation of the Foxo3 downstream target PUMA [66]. Moreover, circRNAs are able to bind and sequester proteins. For example, circMTO1 interacted with TRAF4 by serving as a ceRNA to repress TRAF4 from binding to the $E g 5$ gene, leading to sequester TRAF4 from activating $E g 5$ translation, thus mediating TNBC cell resistance to monastrol [67].

\section{CircRNAs encode proteins}

CircRNAs were previously regarded as a distinct class of endogenous non-coding RNAs that could not translate proteins due to lack of 5-3 polarity, a polyadenylated tail, and an internal ribosome entry site (IRES). However, recent studies indicated that some cytoplasmic circRNAs can be effectively translated into detectable peptides $[68,69]$. IRES- and N[6]-methyladenosines-mediated cap-independent translation initiation have been suggested to be potential mechanism for circRNA translation [70, 71]. To date, several circRNAs have been uncovered to have the potential to be translated into proteins, for instance, circZNF609, circPABPN1 [72, 73]. In TNBC (Fig. 3c), circFBXW7 not only can serve as a sponge of $m i R-197-3 p$ to upregulate its parent gene $F B X W 7$, but also encode the FBXW7$185 \mathrm{aa}$ protein to increase the abundance of FBXW7, thereby promoting $\mathrm{c}-\mathrm{Myc}$ ubiquitination and degradation, eventually suppressing TNBC cells growth and metastasis [74]. More recently, Li et al. confirmed that a newly identified HER2 transcriptional variant, circHER2, had an open reading frame driven by an IRES and could generate a 103 amino acid protein HER2103. HER2-103 could promote homo/hetero dimerization of epidermal growth factor receptor (EGFR)/ HER3 and sustain AKT phosphorylation and downstream malignant phenotype [75]. Whith the increasing evidence prove that circRNAs could translate proteins directly [72, 73, 76, 77], the notion of circRNAs are non-coding RNAs is becoming doubtful.

\section{Role of circRNAs on the biological functions of TNBC}

CircRNAs play an important role in the regulation of cell proliferation, invasion, metastasis, apoptosis, autophagy, cell cycle, vascularization, and chemoresistance of TNBC by regulating the expression of target genes involved in cancer-related signaling pathways directly or indirectly. The biological roles of circRNAs involved in TNBC cells are depicted and summarized in Fig. 4.

\section{CircRNAs modulate TNBC proliferation and tumor growth}

Majority circRNAs identified in TNBC are characterized by oncogenic features. Specifically, circRPPH1, circSEPT9, circGNB1, circPGAP3, circUBE2D2, circRAD18,

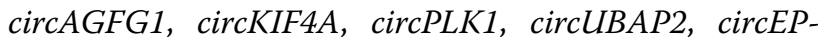
$S T I 1$, and $\operatorname{circGFRA1}$ were upregulated in both TNBC cells and tissues, and high expression of these circRNAs was able to promote tumor cell proliferation both in vitro and in vivo, and was associated with larger tumor sizes and shorter survival times for TNBC patients $[29,46,49$, 51, 53, 54, 57, 59, 60, 63, 64, 78]. Similarly, circZEB1, circEIF3M, circHER2, hsa_circ_0131242, hsa_circ_0005320, hsa_circ_069718, hsa_circ_0058514, and circTFCP2L1 were overexpressed in TNBC cells and tissues, and they appeared to promote cell proliferation and tumor growth of TNBC [55, 58, 62, 75, 79-82]. On the contrary, a few circRNAs were identified to have tumor-suppressive

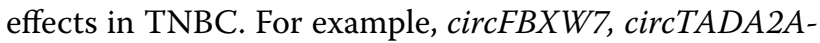
E6, $\operatorname{circITCH}$, and $\operatorname{circAHNAK1}$ were found to be downregulated in TNBC cells and tissues. Their expression was negatively correlated with the tumor sizes and DFS or OS of TNBC patients, and ectopic overexpression of these circRNAs obviously inhibited cell proliferation and tumor growth [48, 52, 56, 74]. Lisewise, $\operatorname{circCDYL}$ was down-regulated in TNBC cells and inhibited proliferation of TNBC cells [61].

\section{CircRNAs affect invasion and metastasis of TNBC}

Certain circRNAs also play pivotal roles in promoting the invasion and metastasis of TNBC. High expression of circSEPT9, circGNB1, circAGFG1, circPGAP3, circKIF4A, $\operatorname{circPLK1}$, $\operatorname{circ} A N K S 1 B$, circUBAP2, and ciRS-7 significantly contributed to the invasion and metastasis of TNBC cells both in vitro and in vivo, and were correlated with advanced TNM stage and poor prognosis of TNBC patients [45, 47, 49, 51, 53, 54, 57, 59, 64]. Likewise, circRPPH1, hsa_circ_0131242, circEIF3M, circHER2, circUBE2D2, circRAD18, hsa_circ_069718, hsa_circ_0058514, and circTFCP2L1 also significantly promoted the migration and invasion capability of TNBC cells in vitro $[55,60,62,63,75,78,79,81,82]$.

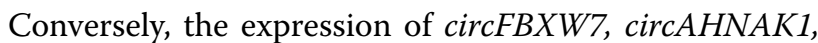




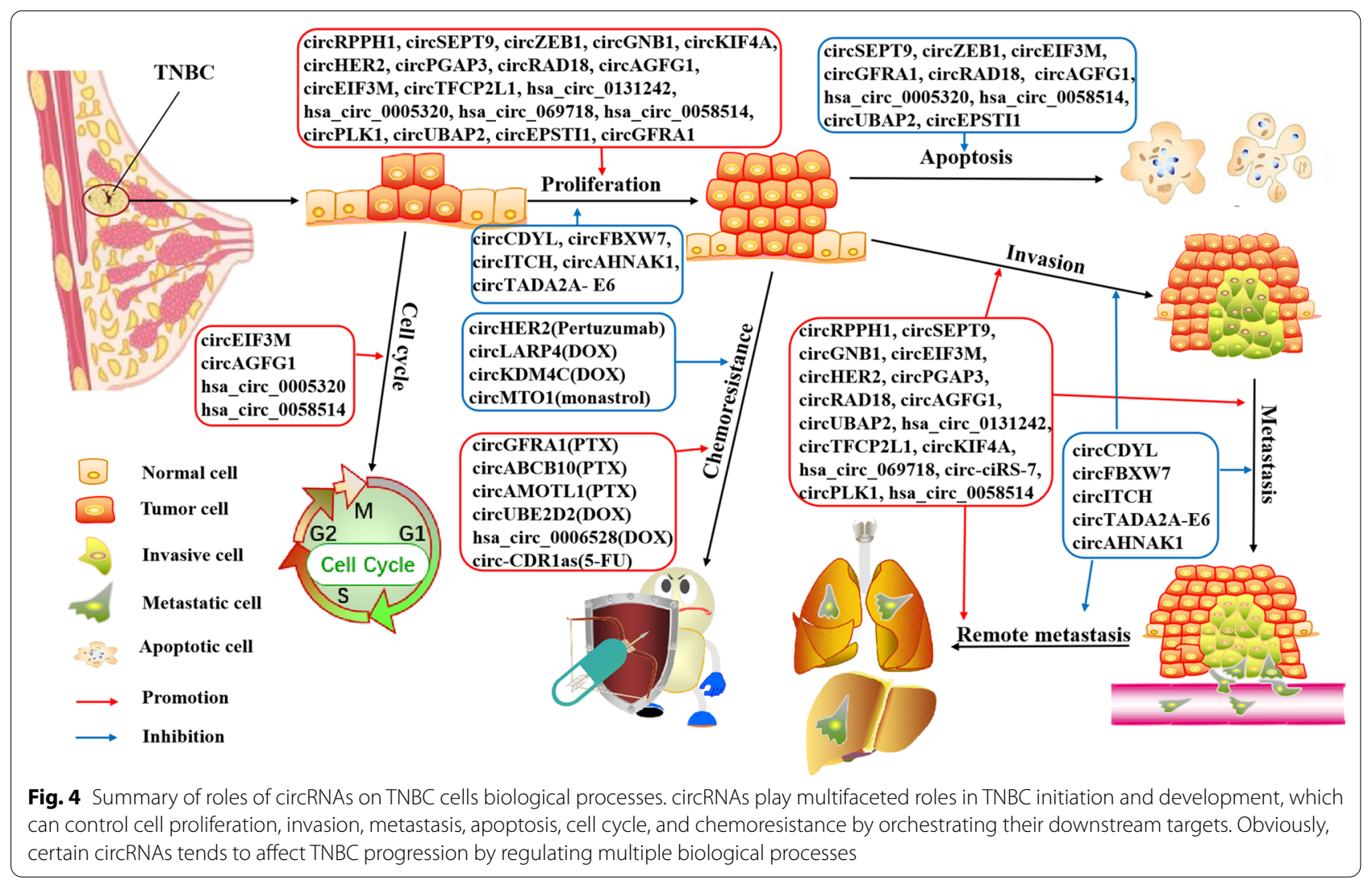

circTADA2A-E6, and circITCH appeared to be downregulated in TNBCs and was associated with advanced TNM stage and poor survival for TNBC patients $[48,52,56$,

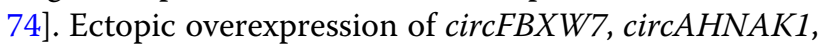
and $\operatorname{circITCH}$ markedly inhibited the migration of TNBC cells in vitro and obviously reduced the size and number of lung metastasis nodules in xenograft models of TNBC [52, 56, 74]. Epithelial-to-mesenchymal transition (EMT) is a process characterized by the loss of the polarity and adhesion capacity of epithelial cells, but an increase in the mesenchymal traits [83],whcih is pivotal for TNBC cells to metastasize [84]. Notably, circRPPH1, hsa_circ_069718, circKIF4A, and circPLK1 could increase the expression of mesenchymal marker vimentin and decrease the expression of epithelial marker E-cadherin, thus contributing to EMT and metastasis [51, 54, 60, 81]. Wnt/ $\beta$-catenin pathway is a key signaling cascade tightly associated with cancer progression. Activation of the Wnt $/ \beta$-catenin pathway could promote tumor invasion by the upregulation of factors regulating the EMT processes [85]. We found that hsa_circ_069718 and circITCH have opposite roles in regulating the $\mathrm{Wnt} / \beta$-catenin pathway. Hsa_circ_069718 activated the Wnt/ $\beta$-catenin pathway by upregulating $\beta$-catenin, $c-M y c$, and cyclin D1 and thus promoted the invasion and metastasis of TNBC cells, while $\operatorname{circITCH}$ played the opposite role [52, 81]. Besides, $\operatorname{circANKS1B}$ was proved to promote EMT via increasing the expression of transcription factor USF1, which could transcriptionally upregulate TGF- $\beta 1$ expression, resulting in activating TGF- $\beta 1 /$ Smad signaling [47]. On the contrary, circTADA2A-E6 exerted a negative effect on the regulation of metastasis by suppressing the EMT process [48]. Above all, these circRNAs might act as potential predictors and therapeutic targets for metastatic TNBC.

\section{CircRNAs regulate apoptosis of TNBC cells}

CircSEPT9, circZEB1, circEIF3M, hsa_circ_0005320, circRAD18, circGFRA1, hsa_circ_0058514, circAGFG1, circUBAP2, and circEPSTI1 have been proven to be upregulated in TNBC cells and tissues, and associated with decreased cell apoptosis rates of TNBC cells $[29,46$, $49,53,57,58,62,78,80,82]$. Silencing of circSEPT9, hsa circ_0005320, or circAGFG1 leads to typical apoptotic morphological characteristics in TNBC cells, such as nuclear shrinkage as well as apoptotic body and nuclear fragmentation $[49,57,80]$. Mechanically, knockdown of circSEPT9, circZEB1, or circAGFG1 could increase the protein levels of the apoptotic markers (cleaved caspase 3 and $B a x$ ) while decrease the anti-apoptotic marker 
(Bcl-2) levels in TNBC cells $[49,57,58]$. Taken together, these circRNAs may mediate the progress of TNBC by suppressing tumor cell apoptosis.

\section{Cell cycle/autophagy/angiogenesis-associated circRNA in TNBC}

It is well known that CCNE1 works by forming a complex with CDK2, and the CCNE1-CDK2 complex is able to pushing cell cycle from $\mathrm{G} 1$ to $\mathrm{S}$ phase, thereby regulating tumor progression [86]. Hsa_circ_0058514 and circAGFG1 were proved significantly up-regulated in TNBC cells and tissues and could promote the CCNE1 and $C D K 2$ expression via acting as miRNAs sponge, the knockdown of hsa_circ_0058514 and circAGFG1 resulted in G1/S phase cell cycle arrest [57, 82]. Similarly, $\operatorname{circEIF3M}$ acts as a ceRNA to upregulate CCND1, which mainly coordinates with cyclin-dependent kinase 4 (CDK4) to regulate cell cycle progression, downregulation of circEIF3M led to G1 arrest [62]. Besides, silencing hsa_circ_0005320 led to higher percentages of TNBC cells being arrested in the G1 phase, with lower percentages of cells in the S phase, suggesting hsa_circ_0005320 also exerts functions in the regulation of the cell cycle of TNBC cells [80]. Meanwhile, Yang et al. also discovered that $\operatorname{circAGFG1}$ significantly promoted tumor angiogenesis, suggesting circAGFG1 also play important roles in the regulation of tumor angiogenesis [57]. Cir$c R P P H 1$ was proved to facilitates angiogenesis in TNBC as well [60]. As for autophagy, the knockdown circSEPT9 in TNBC cells could increase the conversion of the autophagy marker $L C 3$ from $L C 3-I$ to $L C 3-I I$ and upregulate the autophagy-related proteins ATG5 and ATG7, thereby inducing $L C 3 I I$ punctuation and accumulation of autophagosomes [49].

\section{CircRNAs regulate TNBC resistance to therapeutic drugs}

Chemotherapy is a critical strategy for TNBC treatment, which is usually administered as postoperative therapy or preoperative neoadjuvant therapy. The most commonly used chemotherapeutics, including anthracyclines (doxorubicin[DOX] and epirubicin), taxanes (PTX and docetaxel), 5-fluorouracil (5-FU), and cyclophosphamide, have achieved substantial advantages for TNBC patients, but do not work out for every patient due to drug resistance [4]. CircRNAs have been reported to play vital roles in drug resistance, either by promoting or reversing TNBC chemoresistance [87].

DOX-based chemotherapy is the most frequently used treatment for TNBC [88]. Hsa_circ_0006528,

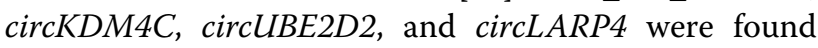
to be associated with DOX resistance in TNBC [63, 87, 89, 90]. Specifically, hsa_circ_0006528 was significantly upregulated in DOX-resistant MDA-MB-231
(MDA-MB-231/DOX) cells [87], mechanically by participating in the circ_0006528/miR-7-5p/Raf1 axis that confers chemotherapeutic resistance in TNBC [91]. Likewise, circUBE2D2 decreased DOX-induced TNBC cells apoptosis by upregulating CDCA3, which is a trigger of mitotic entry to withstand the DOX-induced apoptosis, indicating that circUBE2D2 promotes DOX resistance of TNBC cell [63]. Instead, circLARP4 was downregulated in TNBC cell lines, and ectopic overexpression of circLARP4 can increase the sensitivity of MDA-MB-231 cell lines to DOX [89]. In addition, circKDM4C experession was significantly decreased in MDA-MB-231/DOX cells and could attenuate DOX resistance by upregulating $P B L D$ [90], which is a tumor suppesssor that could inhibit tumor growth [92].

Chemoresistance against PTX is one of the major issues related to treatment failure in TNBC patients. However, the mechanism by which TNBC cells become resistant to PTX remains unclear. Recently, three circRNAs, CircGFRA1, circABCB10 and circAMOTL1, were identified as important factors that may be responsible for the adverse resistance to PTX in TNBC cells. Specifically, $\operatorname{CircGFRA1}$ and $\operatorname{circABCB10}$ were upregulated in the PTX-resistant MDA-MB-231 (MDA-MB-231/ PTX) cells. CircGFRA1 knockdown can inhibit the resistance of TNBC cells to PTX by reducing the expression of TLR4, which has been found to be activated by paclitaxel to improve tumor cell survival and blocking TLR4 could significantly improve response to paclitaxel therapy in BC $[50,93]$. CircABCB10 contributed to PTX resistance of TNBC cells through up-regulating of DUSP7, which exerts its function by dephosphorylating MAPK [94, 95]. Besides, circAMOTL1 promoted the chemoresistance against PTX in TNBC cells via posttranscriptional regulation of $\mathrm{AKT}$ and therefore led to increase the anti-apoptotic gene $B C L 2$ expression and inhibit the proapoptotic gene $B A X$ and $B A K$ expression [96]].

Moreover, circ-CDR1as was found to be associated with 5-FU-resistant in MDA-MB-231 cells by inhibiting miR-7 to upregulate CCNE1 [97]. Besides, circMTO1, which is usually downregulated in monastrol-resistant MDA-MB-231 cells, can promote monastrolinduced cytotoxicity by targeting Eg5 and sequestering TRAF4 from binding to the Eg5 gene [67]. Interestingly, circHER2, which encodes a novel protein HER2-103, was proved to be expressed in some TNBC samples, and HER2-103-positive TNBC cells were sensitive to Pertuzumab due to HER2-103 shared the same amino acid sequences as the HER2 CR1 domian [75].

More and more circRNAs are being identified to be associated with chemoresistance, however, our understanting of the mechanistic role of circRNAs contributing to chemotherapeutic resistance is limited due to 
lack of deep mechanistic investigations and in vivo studies. Whether circRNAs could be a potential target for overcoming TNBC chemoresistance requires further exploration.

\section{Clinical significance of circRNAs in TNBC}

CircRNAs have also been proven to possess potential values for diagnosis and prognosis of TNBC. As a result, circRNAs have received considerable interest for their potential as prognostic markers or therapeutic targets.

\section{CircRNAs acts as diagnostic biomarkers for TNBC}

There are six circRNAs have been currently identified to have diagnostic values in TNBC. Among them, three circRNAs were upregulated and the other three were downregulated in TNBC (Table 2). CircAHNAK1 was the first identified circRNA that significantly downregulated in TNBC tissues, therefore it can be used as a diagnostic indicator for distinguishing TNBC from normal breast tissue [56]. Xu et al. [48] identified two differentially expressed circTADA2As, circTADA2A-E6 and circTADA2A-E5/E6, that were spliced from exon 6 or exons 5 and 6 of the same TADA2A gene respectively, in TNBC, and found that these two circRNAs were significantly downregulated in TNBC and exhibited excellent

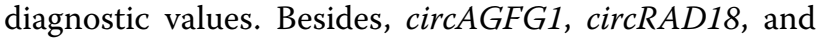
circSEPT9 were found to be upregulated in TNBC and also exhibited excellent ability in discriminating between TNBC patients and normal individuals $[49,57,78]$.

\section{Association of circRNAs with clinicopathological features of TNBC}

Based on the current reported evidences, we summarized the relationship between circRNAs expression and the clinicopathological factors of TNBC in Additional file 1: Tables S1 and S2. Accordingly, there was no significant correlation between any circRNAs expression and age or menopause status of TNBC patients (Additional file 2: Figure 1). While many circRNAs expression was significantly associated with other clinical parameters of TNBC, including tumor size, lymph node metastasis, histological grade, and TNM stage (Table 3). More impor-

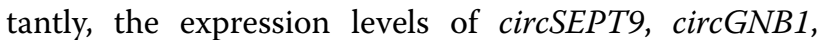
hsa_circ_0131242, circPGAP3, circRAD18, circAGFG1,

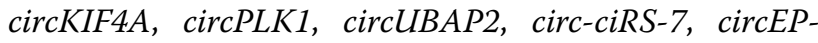
$S T I 1$, and $\operatorname{circGFRA1}$ were positively correlated with the tumor size $[29,45,46,49,51,53,54,57,59,60,64,78$,

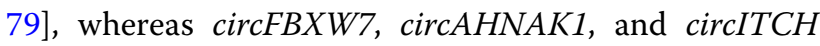
presented negative associations (Fig. 5a) [52, 56, 74]. CircAGFG1 and $\operatorname{circGFRA1}$ were positively related to

Table 2 Summary of diagnosis values of circRNAs in TNBC

\begin{tabular}{|c|c|c|c|c|c|c|c|c|c|}
\hline CircRNAs & TNBC samples & $\begin{array}{l}\text { Normal } \\
\text { samples }\end{array}$ & $\begin{array}{l}\text { Expression } \\
\text { in TNBC }\end{array}$ & Method & Sample type & $A \cup C$ & Sensitivity & Specificity & References \\
\hline circSEPT9 & 60 & 60 & Up & RT-qPCR & Tissue & 0.711 & 0.633 & 0.75 & [49] \\
\hline circRAD18 & 31 & 31 & Up & RT-qPCR & Tissue & 0.752 & NR & NR & {$[78]$} \\
\hline $\operatorname{circAGFG1}$ & 40 & 40 & Up & RT-qPCR & Tissue & 0.767 & $N R$ & $N R$ & {$[57]$} \\
\hline circTADA2A-E6 & 115 & 16 & Down & $R T-q P C R$ & Tissue & 0.8554 & $N R$ & $N R$ & {$[48]$} \\
\hline circTADA2A-E5/E6 & 115 & 16 & Down & RT-qPCR & Tissue & 0.9366 & $N R$ & $N R$ & {$[48]$} \\
\hline circAHNAK1 & 20 & 20 & Down & RT-qPCR & Tissue & 0.72 & NR & $N R$ & {$[56]$} \\
\hline
\end{tabular}

$T N B C$ triple-negative breast cancer, $N R$ not report, $q R T-P C R$ quantitative real-time $P C R, A U C$ area under the curve

Table 3 Summary of circRNAs related to clinicopathological features of TNBC

\begin{tabular}{|c|c|c|}
\hline \multirow[t]{2}{*}{ Clinicopathological factors } & \multicolumn{2}{|c|}{ Correlation of circRNAs expression with clinicopathological factors in TNBC } \\
\hline & Positive & Negative \\
\hline Tumor size & $\begin{array}{l}\text { circSEPT9, circGNB1, hsa_circ_0131242,circPGAP3, } \\
\text { circRAD18, circAGFG1, circKIF4A, circPLK1, circUBAP2, circ- } \\
\text { ciRS-7, circEPSTI1, circGFRA1 }\end{array}$ & circFBXW7, circAHNAK1, circlTCH \\
\hline Histological grade & circAGFG1, circGFRA1 & NR \\
\hline LN metastasis & $\begin{array}{l}\text { circRPPH1, circSEPT9, circPGAP3, circUBE2D2, circAGFG1, } \\
\text { circKIF4A, circPLK1, circANKS1B, circUBAP2, circ-ciRS-7, } \\
\text { circEPST11, circGFRA1 }\end{array}$ & circUSP42, circFBXW7,circTADA2A-E6,circAHNAK1,circlTCH \\
\hline TNM stage & $\begin{array}{l}\text { circSEPT9, circGNB1, hsa_circ_0131242, circPGAP3, } \\
\text { circUBE2D2, circRAD18, hsa_circ_069718, circKIF4A, } \\
\text { circPLK1,circANKS1B, circUBAP2, circEPSTI1 }\end{array}$ & circUSP42, circTADA2A-E6,circAHNAK1, circITCH \\
\hline
\end{tabular}




\begin{tabular}{|c|c|c|}
\hline a & pvalue & \\
\hline Yehui Zhou 2020 - circRPPH1 & 0.409 & $3.333(0.515-21.584)$ \\
\hline Xiaying Zheng 2020 - circSEPT9 - Cohort 1 & 0.002 & $6.000(1.890-19.043)$ \\
\hline Xiaying Zheng 2020 - circSEPT9 - Cohort 2 & 0.009 & $3.483(1.338-9.07$ \\
\hline Peng Liu 2020 - circGNB1 & 0.001 & $4.735(2.184-10.264)$ \\
\hline Yueting Li 2020 - hsa_circ_0131242 & $<0.001$ & $5.296(1.993-14.074$ \\
\hline Dabao He 2020 - circ-PGAP3 & 0.008 & $3.345(1.346-8.312$ \\
\hline Yutian Zou 2019 - circRAD18 & 0.002 & $4.293(1.675-11.001)$ \\
\hline Yutian Zou 2019* - circRAD18 & 0.001 & 4.523(1.844-11.093) \\
\hline Rui Yang 2019 - circAGFG1 - Cohort 1 & 0.027 & $4.333(1.150-16.323)$ \\
\hline Rui Yang 2019 - circAGFG1 - Cohort 2 & 0.152 & $2.000(0.769-5.198)$ \\
\hline Feng Ye 2019 - circFBXW7 & 0.014 & $0.593(0.390-0.900)$ \\
\hline Weikai Xiao 2019*- circAHNAK1 & 0.045 & $0.239(0.064-0.900)$ \\
\hline S. T. WANG 2019 - circITCH & 0.016 & $0.354(0.150-0.831)$ \\
\hline Qian Wang 2019 - circTFCP2L1 & 0.154 & $3.545(0.592-21.245)$ \\
\hline Hailin Tang 2019 - circKIF4A & $<0.001$ & $3.247(1.699-6.207)$ \\
\hline Yanan Kong 2019 - circPLK1 & $<0.001$ & $3.984(2.028-7.829)$ \\
\hline Kaixuan Zeng 2018 - circANKS1B & 0.180 & $0.626(0.314-1.245)$ \\
\hline Shengting Wang 2018 - circUBAP2 & 0.003 & $0.240(0.092-0.627)$ \\
\hline Meixiang Sang $2018^{\star}$ - circ-ciRS-7 & 0.005 & $3.935(1.452-10.666)$ \\
\hline Bo Chen 2018 - circEPSTI1 & $<0.001$ & $6.536(2.825-15.123$ \\
\hline Rongfang He 2017 - circGFRA 1 & 0.029 & $1.884(1.062-3.340)$ \\
\hline \multicolumn{3}{|l|}{ C } \\
\hline Study & pvalue & Odds ratio \\
\hline Yehui Zhou 2020 - circRPPH1 & 0.025 & $9.333(1.193-72.991)$ \\
\hline Xiaying Zheng 2020 - circSEPT9 & 0.028 & $3.500(1.112-11.017)$ \\
\hline Jinling Yu 2020 - circUSP42 & 0.005 & $0.063(0.006-0.604)$ \\
\hline Peng Liu 2020 - circGNB1 & 0.072 & $1.652(0.954-2.860)$ \\
\hline Dabao He 2020 - circ-PGAP3 & $<0.001$ & $6.496(2.369-17.815)$ \\
\hline Yutian Zou 2019 - circRAD18 & 0.109 & $1.779(0.878-3.604)$ \\
\hline Feng Ye 2019 - circFBXW7 & 0.004 & $0.578(0.398-0.839)$ \\
\hline Rui Yang 2019 - circAGFG1 + Cohort 1 & 0.025 & $4.500(1.166-17.373)$ \\
\hline Rui Yang 2019 - circAGFG1 + Cohort 2 & 0.260 & $1.667(0.684-4.063)$ \\
\hline Weikai Xiao 2019 - circAHNAK1 & 0.001 & $0.274(0.135-0.560)$ \\
\hline S. T. WANG 2019 - circlTCH & 0.008 & $0.315(0.132-0.753)$ \\
\hline Hailin Tang 2019 - circkIF $4 A$ & $<0.001$ & $2.712(1.597-4.606)$ \\
\hline Yanan Kong 2019 - circPLK1 & $<0.001$ & $2.620(1.543-4.449)$ \\
\hline Kaixuan Zeng 2018 - circANKS1B & 0.004 & $2.510(1.336-4.714)$ \\
\hline Shengting Wang 2018 - circUBAP2 & 0.002 & $0.221(0.081-0.601)$ \\
\hline Meixiang Sang 2018 - circ-ciRS-7 & 0.028 & $2.155(1.079-4.306)$ \\
\hline Bo Chen 2018 - circEPSTI1 & 0.004 & $2.197(1.276-3.785)$ \\
\hline Rongfang He 2017 - circGFRA1 & $<0.001$ & $16.131(8.214-31.678)$ \\
\hline
\end{tabular}
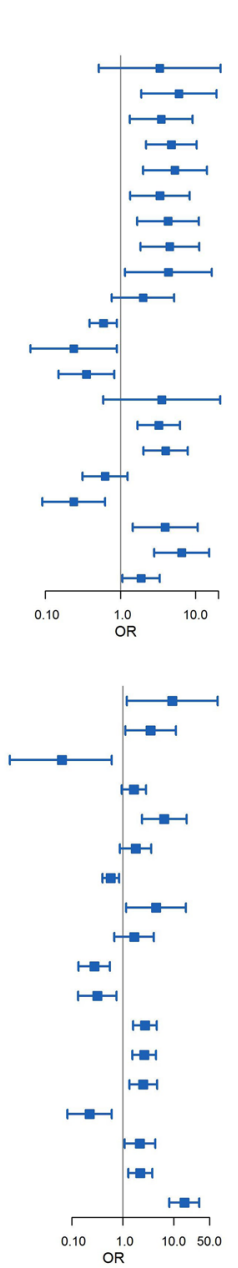

b

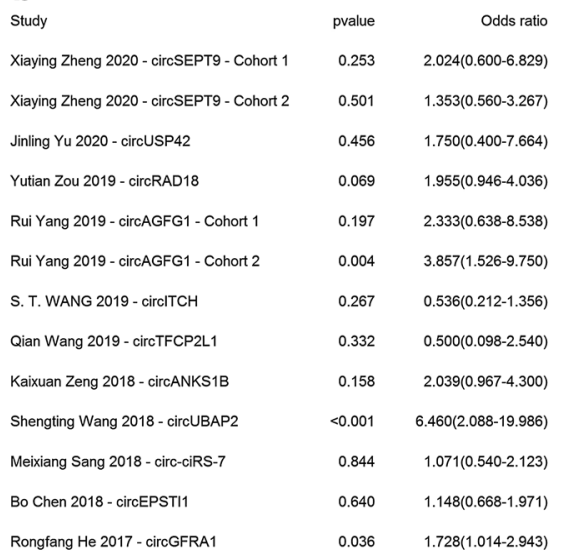

d

Ytudy Zhou 2020 - circRPPH1

Xiaying Zheng 2020" - circSEPT9 - Cohort 1

Xiaying Zheng 2020" - circSEPT9 - Cohort 2

Jinling Yu 2020 - circUSP42

Peng Liu 2020 - circGNB1

Yueting Li 2020" - hsa_circ_0131242

Dabao He 2020 - circ-PGAP3

Yutian Zou 2019 - circRAD18

Feng Ye 2019 - dircFBXW7

Rui Yang 2019 - circAGFG1 - Cohort 1

Rui Yang 2019 - circAGFG1 - Cohort 2

Weikai Xia 2019 - dirCAHNAK1

S. T. WANG 2019 - circlTCH

Qian Wang 2019 - circTFCP2L1

Hailin Tang 2019 - circkIF4A

Yanan Kong 2019 - circPLK1

Kaixuan Zeng 2018 - circANKS1B

Shengting Wang 2018 - circUBAP2

Bo Chen 2018 - circEPST11

Rongfang He 2017 - circGFRA1

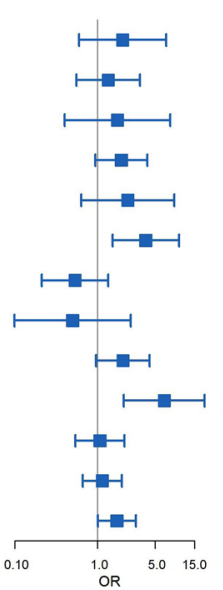

pvalue Odds ratio

$0.068 \quad 6.000(0.812-44.351)$

$0.001 \quad 7.429(2.078-26.553)$

$5.935(1.744-20.200)$

1.364(1.005-1.850)

2.368(1.248-4.493)

$6.000(2.332-15.436)$

5.472(1.800-16.637)

2.909(1.274-6.642)

$0.818(0.558-1.198)$

$3.000(0.507-17.740)$

$1.615(0.614-4.247)$

$0.107(0.035-0.331)$

$0.163(0.054-0.487)$

7.286(1.173-45.255)

2.180(1.174-4.047)

2.231(1.201-4.143)

2.826(1.431-5.580)

4.714(1.611-13.796)

$2.298(1.229-4.299)$

8.550(4.060-18.007)

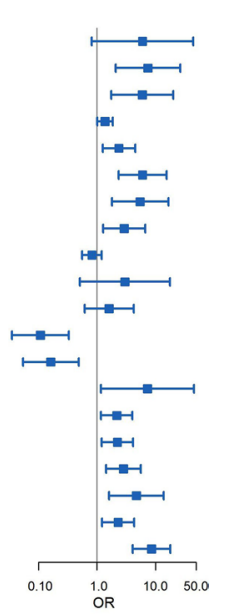

Fig. 5 Forest plots of the associations between the expression of circRNAs and $\mathbf{a}$ tumor sizes ( $>2$ vs $\leq 2)$, $\mathbf{b}$ histological grade (III vs $I+I I)$, $\mathbf{c l y m p h}$ node metastasis (positive vs negative), d TNM stage (III + IV vs I+II) of TNBC. Each square indicates a study. * indicates $>5$ versus $\leq 5$, \# indicates III + II versus I; OR odds ratio, Cl confidence interval

the histological grade of TNBC (Fig. 5b) [29, 57]. High expressions of circRPPH1, circSEPT9, $\operatorname{circAGFG1,~} \operatorname{circP}$ -

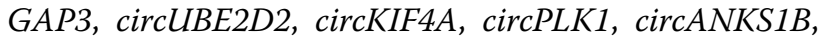
circUBAP2, circ-ciRS-7, circEPSTI1, and circGFRA1 were associated with positive lymph node metastasis $[29,45-47,51,53,54,57,60,63,64]$, while low expressions of circUSP42, circFBXW7, circTADA2A-E6, circAHNAK1, and $\operatorname{circITCH}$ were associated with positive lymph node metastasis in TNBC (Fig. 5c) [48, 52, 56, 74]. Besides, increased expression of circSEPT9, circGNB1, hsa_circ_0131242, circPGAP3, circUBE2D2, circRAD18, hsa_circ_069718, circKIF4A, circPLK1, circANKS1B, circUBAP2, and circEPSTI1 in TNBC suggested advanced TNM stage $[46,47,49,51,53,54,59,63,64$, $78,79,81]$, whereas decreased expression of circUSP42,
circTADA2A-E6, circAHNAK1, and circITCH in TNBC indicated advanced TNM stage (Fig. 5d) [48, 52, 56].

\section{CircRNAs act as prognostic biomarkers for BC}

21 circRNAs have been reported to have the prognostic values for TNBC patients (Table 4). Specifically, high expression of circSEPT9, $\operatorname{circGNB1}$, circRAD18, $\operatorname{circAGFG1}$, and $\operatorname{circANKS1B}$ and low expression of circFBXW7 were related to worse survival of TNBC and could be independent prognostic factors for TNBC patients $[47,49,57,59,74,78]$, while $\operatorname{circTADA2A-E6}$ was frequently downreglated in TNBC and whose downregulation were associated with worse survival (Fig. 6). High expression of 15 circRNAs ( $\operatorname{circSEPT9}$, circGNB1, hsa_circ_0131242, circHER2, circPGAP3, circUBE2D2, circRAD18, circAGFG1, hsa_circ_069718, circKIF4A, 


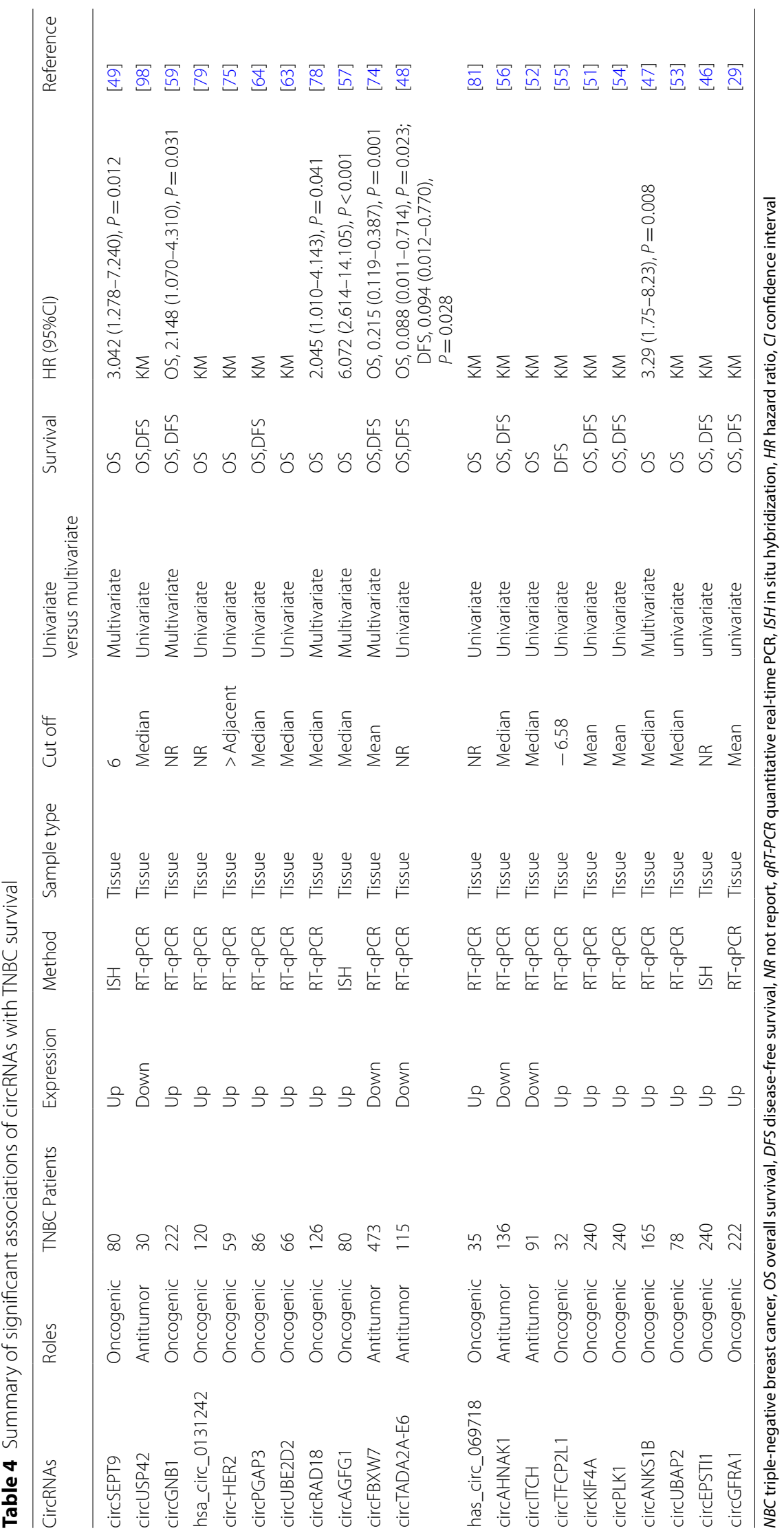




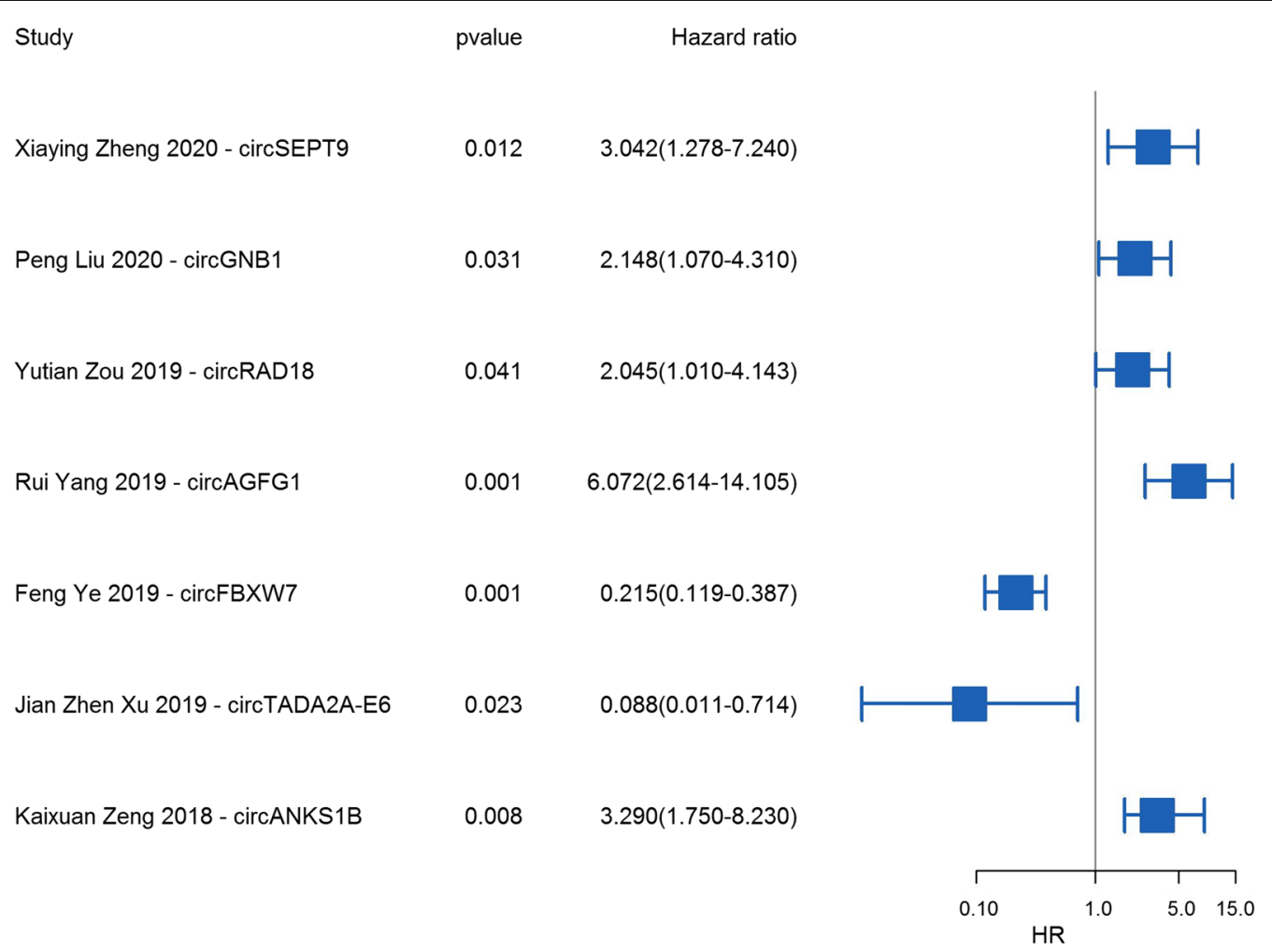

Fig. 6 Forest plots of the associations between the expression of circRNAs and TNBC overall survival. Each square indicates a study. HR hazard ratio, Cl confidence interval

circPLK1, $\operatorname{circANKS1B,~} \operatorname{circUBAP2,} \operatorname{circEPSTI1,~and~}$ circGFRA1) was related to worse OS $[46,47,49,51,53$, $54,57,59,63,64,75,78,79,81]$, indicating that they have carcinogenic effects in TNBC. Increased expression of the 5 circRNAs (circLSP42, circFBXW7, circTADA2A-E6, circAHNAK1, and $\operatorname{circITCH)}$ was associated with better OS for TNBC patients [48, 52, 56, 74, 98], suggesting that they serve as tumor suppressors. In terms of DFS, elevated expression of 7 circRNAs (circGNB1, circPGAP3,

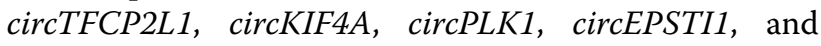
circGFRA1) showed reduced DFS [29, 46, 51, 54, 55, 59,

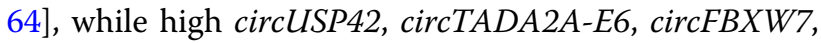
and $\operatorname{circAHNAK1}$ expression predicted better DFS [48, $56,74,98]$, implying that they are related to the recurrence or progression of TNBC.

\section{Conclusions and perspective}

TNBC represents a more malignant and aggressive subtype of $\mathrm{BC}$ that lacks of effective targeted therapies, and the specific pathogenesis of TNBC is still not fully understood. CircRNAs, previously thought to be the products of RNA splicing errors, are now regarded as an emerging vital player with intriguing functions during various physiological and pathophysiological processes. As discussed in this review, the exact mechanisms of circRNAs maturation have not been fully elucidated, while the dysregulation of circRNAs is believed to be one of the important mechanisms leading to the development and progression of TNBC. As regulators of gene expression, circRNAs are involved in various biological processes of TNBC, including cell proliferation, apoptosis, cell cycle, angiogenesis, metastasis and chemoresistance, making them potential promising biomarkers for TNBC in regarding to diagnosis, prognosis or treatment.

Besides, there are several gaps in the research field of circRNA in TNBC, which need to be further fulfilled in the future. CircRNAs are always maintained at a relatively stable state in cells and they live long in the extracellular environment owing to their unique structures that are resistant to exonuclease RNase Rand [32, 99], identification of dysregulated circRNAs in body fluids, therefore, may be more beneficial for the diagnosis and prognosis of TNBC. Currently, circRNAs in clinical samples, such as plasma, serum, or exosomes have been found to be serve as significant biomarkers in tumor. For example, Wang et al. have identified and validated a number of dysregulated circRNAs in exosomes from $\mathrm{BC}$ patients [100] and circUBE2D2 was found to 
significantly load in exosomes isolated from tamoxifenresistant cells, which reinforced tamoxifen resistance in BC [101]. Nevertheless, there is no literature reporting the circulating circRNAs (from blood, urine, saliva, etc.) in TNBC, which should be pursued in future researches. Additionally, the well-known mechanism of circRNAs exerting functions in biological or pathological processes is through ceRNA to target downstream genes, while other potent molecular mechanisms of circRNAs involved in TNBC progression is limited and needs further investigation. Moreover, other unexcavated circRNAs related to TNBC development and progression and elucidation of their corresponding functions are also awaiting for discovery.

Overall, this review gives a systematically summary of the biogenesis, regulatory mechanisms, and biological functions of circRNAs in TNBC, and lists almost all of the circRNAs that dysregulated in TNBC and discusses their significant values for TNBC in regarding to diagnosis, prognosis and chemoresistance, which provides great guiding significance for future researches of circRNAs in TNBC. A better understanding of circRNAs in TNBC may contribute to the development of more reliable diagnosis and treatment straategies for TNBC.

\begin{abstract}
Abbreviations
BC: Breast cancer; TNBC: Triple-negative breast cancer; ER: Estrogen receptor; PR: Progesterone receptor; HER2: Human epidermal growth factor receptor 2; TAMs: Tumor-associated macrophages; miRNAs: MicroRNAs; IncRNAs: Long non-coding RNA; circRNAs: Circular RNAs; RNA-seq: RNA sequencing; qRT-PCR: Quantitative real-time PCR; pre-mRNAs: Precursor messenger RNAs; EcircRNAs: Exon circRNAs; ciRNAs: Circular intronic RNAs; ElciRNAs: Exon-intron circRNAs; f-circRNAs: Fusion circRNAs; RBPs: RNA-binding proteins; ceRNAs: Competing endogenous RNAs; mRNAs: Messenger RNAs; IRES: Internal ribosome entry site; EMT: Epithelial-to-mesenchymal transition; DOX: Doxorubicin; MDAMB-231/DOX: DOX resistant MDA-MB-231; PTX: Paclitaxel; MDA-MB-231/PTX: PTX resistant MDA-MB-231; 5-FU: 5-Fluorouracil; OS: Overall survival; DFS: Disease-free survival.
\end{abstract}

\section{Supplementary Information}

The online version contains supplementary material available at https://doi. org/10.1186/s13045-021-01052-y.

Additional file 1. Summary of the relationship between circRNAs expression and the clinicopathological factors of TNBC.

Additional file 2. Forest plots of the associations between the expression of circRNAs and $\mathbf{a}$ age (older vs young), $\mathbf{b}$ menopause (Yes vs No) of TNBC patients. Each square indicates a study.

\section{Acknowledgements}

We thank all members of our study team for their wonderful cooperation.

\section{Authors' contributions}

$L J, S Z Z, M W$ collected the related paper. $L L$ and XY D drafted and wrote the manuscript. SZ Z and YJ D revised the manuscript. ZJ D, YW, and SY participated in the design of the review and helped to draft and revise the manuscript. All authors read and approved the final manuscript.
Funding

This study was funded by none institution or individual.

Availability of data and materials

Not applicable.

\section{Declarations}

Ethics approval and consent to participate

Not applicable.

\section{Consent for publication}

Not applicable.

\section{Competing interests}

The authors declare that they have no competing interests.

\section{Author details}

${ }^{1}$ Department of Breast Surgery, The First Affiliated Hospital, College of Medicine, Zhejiang University, Hangzhou 310003, China. ${ }^{2}$ Department of Oncology, The Second Affiliated Hospital of Xi'an Jiaotong University, Xi'an, China. ${ }^{3}$ The Cancer Institute of the Second Affiliated Hospital and Institute of Translational Medicine, Zhejiang University School of Medicine, Hangzhou, China.

Received: 17 December 2020 Accepted: 25 February 2021

Published online: 06 March 2021

\section{References}

1. Li N, Deng Y, Zhou L, Tian T, Yang S, Wu Y, et al. Global burden of breast cancer and attributable risk factors in 195 countries and territories, from 1990 to 2017: results from the Global Burden of Disease Study 2017. J Hematol Oncol. 2019;12(1):140.

2. Ahmad A. Breast cancer statistics: recent trends. Adv Exp Med Biol. 2019;1152:1-7.

3. Lehmann BD, Pietenpol JA, Tan AR. Triple-negative breast cancer: molecular subtypes and new targets for therapy. Am Soc Clin Oncol Educ Book Am Soc Clin Oncol Annu Meet. 2015;35:e31-9.

4. Abramson VG, Lehmann BD, Ballinger TJ, Pietenpol JA. Subtyping of triple-negative breast cancer: implications for therapy. Cancer. 2015;121(1):8-16.

5. Li X, Yang J, Peng L, Sahin AA, Huo L, Ward KC, et al. Triple-negative breast cancer has worse overall survival and cause-specific survival than non-triple-negative breast cancer. Breast Cancer Res Treat. 2017;161(2):279-87.

6. Hwang SY, Park S, Kwon Y. Recent therapeutic trends and promising targets in triple negative breast cancer. Pharmacol Ther. 2019;199:30-57.

7. Eroles P, Bosch A, Perez-Fidalgo JA, Lluch A. Molecular biology in breast cancer: intrinsic subtypes and signaling pathways. Cancer Treat Rev. 2012;38(6):698-707.

8. Mei J, Hao L, Wang H, Xu R, Liu Y, Zhu Y, et al. Systematic characterization of non-coding RNAs in triple-negative breast cancer. Cell Prolif. 2020;53:e12801.

9. Sanger HL, Klotz G, Riesner D, Gross HJ, Kleinschmidt AK. Viroids are single-stranded covalently closed circular RNA molecules existing as highly base-paired rod-like structures. Proc Natl Acad Sci USA. 1976;73(11):3852-6.

10. Capel B, Swain A, Nicolis S, Hacker A, Walter M, Koopman P, et al. Circular transcripts of the testis-determining gene Sry in adult mouse testis. Cell. 1993;73(5):1019-30.

11. Pasman Z, Been MD, Garcia-Blanco MA. Exon circularization in mammalian nuclear extracts. RNA (New York, NY). 1996;2(6):603-10.

12. Ashwal-Fluss R, Meyer M, Pamudurti NR, Ivanov A, Bartok O, Hanan M, et al. circRNA biogenesis competes with pre-mRNA splicing. Mol Cell. 2014;56(1):55-66.

13. Wang PL, Bao Y, Yee MC, Barrett SP, Hogan GJ, Olsen MN, et al. Circular RNA is expressed across the eukaryotic tree of life. PLoS ONE. 2014;9(6):e90859.

14. JeckWR, Sharpless NE. Detecting and characterizing circular RNAs. Nat Biotechnol. 2014;32(5):453-61. 
15. Chaabane M, Andreeva K, Hwang JY, KookTL, Park JW, Cooper NGF. seekCRIT: detecting and characterizing differentially expressed circular RNAs using high-throughput sequencing data. PLoS Comput Biol. 2020;16(10):e1008338.

16. Hansen TB, Venø MT, Damgaard CK, Kjems J. Comparison of circular RNA prediction tools. Nucleic Acids Res. 2016;44(6):e58.

17. Zhou R, Wu Y, Wang W, Su W, Liu Y, Wang Y, et al. Circular RNAs (circRNAs) in cancer. Cancer Lett. 2018;425:134-42.

18. Memczak S, Jens M, Elefsinioti A, Torti F, Krueger J, Rybak A, et al. Circular RNAs are a large class of animal RNAs with regulatory potency. Nature. 2013;495(7441):333-8

19. Lei K, Bai H, Wei Z, Xie C, Wang J, Li J, et al. The mechanism and function of circular RNAs in human diseases. Exp Cell Res. 2018;368(2):147-58.

20. Chen LL. The expanding regulatory mechanisms and cellular functions of circular RNAs. Nat Rev Mol Cell Biol. 2020;21(8):475-90.

21. Tang Q, Hann SS. Biological roles and mechanisms of circular RNA in human cancers. OncoTargets Therapy. 2020;13:2067-92.

22. Shan C, Zhang Y, Hao X, Gao J, Chen X, Wang K. Biogenesis, functions and clinical significance of circRNAs in gastric cancer. Mol Cancer. 2019;18(1):136

23. Marotti JD, de Abreu FB, Wells WA, Tsongalis GJ. Triple-negative breast cancer: next-generation sequencing for target identification. Am J Pathol. 2017;187(10):2133-8.

24. Li Z, Chen Z, Hu G, Zhang Y, Feng Y, Jiang Y, et al. Profiling and integrated analysis of differentially expressed circRNAs as novel biomarkers for breast cancer. J Cell Physiol. 2020;235:7945-59.

25. Nair AA, Niu N, Tang X, Thompson KJ, Wang L, Kocher JP, et al. Circular RNAs and their associations with breast cancer subtypes. Oncotarget. 2016;7(49):80967-79.

26. Yuan C, Zhou L, Zhang L, Yin K, Peng J, Sha R, et al. Identification and integrated analysis of key differentially expressed circular RNAs in ERpositive subtype breast cancer. Epigenomics. 2019;11(3):297-321.

27. Zhou SY, Chen W, Yang SJ, Xu ZH, Hu JH, Zhang HD, et al. The emerging role of circular RNAs in breast cancer. Biosci Rep. 2019;39(6):1.

28. Geng X, Jia Y, Zhang Y, Shi L, Li Q, Zang A, et al. Circular RNA: biogenesis, degradation, functions and potential roles in mediating resistance to anticarcinogens. Epigenomics. 2020;12(3):267-83.

29. He R, Liu P, Xie X, Zhou Y, Liao Q, Xiong W, et al. circGFRA1 and GFRA1 act as ceRNAs in triple negative breast cancer by regulating miR-34a. J Exp Clin Cancer Res CR. 2017;36(1):145.

30. Kristensen LS, Andersen MS, Stagsted LVW, Ebbesen KK, Hansen TB, Kjems J. The biogenesis, biology and characterization of circular RNAs. Nat Rev Genet. 2019;20(11):675-91.

31. Li X, Yang L, Chen LL. The biogenesis, functions, and challenges of circular RNAs. Mol Cell. 2018;71(3):428-42.

32. Li Y, Zheng Q, Bao C, Li S, Guo W, Zhao J, et al. Circular RNA is enriched and stable in exosomes: a promising biomarker for cancer diagnosis. Cell Res. 2015;25(8):981-4.

33. Shang Q, Yang Z, Jia R, Ge S. The novel roles of circRNAs in human cancer. Mol Cancer. 2019;18(1):6.

34. Gao Y, Wang J, Zheng Y, Zhang J, Chen S, Zhao F. Comprehensive identification of internal structure and alternative splicing events in circular RNAs. Nat Commun. 2016;7:12060.

35. Li Z, Huang C, Bao C, Chen L, Lin M, Wang X, et al. Exon-intron circular RNAs regulate transcription in the nucleus. Nat Struct Mol Biol. 2015;22(3):256-64.

36. Meng S, Zhou H, Feng Z, Xu Z, Tang Y, Li P, et al. CircRNA: functions and properties of a novel potential biomarker for cancer. Mol Cancer. 2017;16(1):94.

37. Zhang Y, Zhang XO, Chen T, Xiang JF, Yin QF, Xing YH, et al. Circular intronic long noncoding RNAs. Mol Cell. 2013;51(6):792-806.

38. Gao Y, Wang J, Zhao F. CIRl: an efficient and unbiased algorithm for de novo circular RNA identification. Genome Biol. 2015;16(1):4.

39. Qu S, Yang X, Li X, Wang J, Gao Y, Shang R, et al. Circular RNA: a new star of noncoding RNAs. Cancer Lett. 2015;365(2):141-8.

40. Bartel DP. MicroRNAs: target recognition and regulatory functions. Cell. 2009;136(2):215-33.

41. Tay Y, Rinn J, Pandolfi PP. The multilayered complexity of ceRNA crosstalk and competition. Nature. 2014;505(74):344-52.
42. Hansen TB, Jensen TI, Clausen BH, Bramsen JB, Finsen B, Damgaard CK, et al. Natural RNA circles function as efficient microRNA sponges. Nature. 2013;495(7441):384-8.

43. Han C, Seebacher NA, Hornicek FJ, Kan Q, Duan Z. Regulation of microRNAs function by circular RNAs in human cancer. Oncotarget. 2017;8(38):64622-37.

44. Verduci L, Strano S, Yarden Y, Blandino G. The circRNA-microRNA code: emerging implications for cancer diagnosis and treatment. Mol Oncol. 2019;13(4):669-80.

45. Sang M, Meng L, Liu S, Ding P, Chang S, Ju Y, et al. Circular RNA ciRS-7 maintains metastatic phenotypes as a ceRNA of miR-1299 to target MMPs. Mol Cancer Res MCR. 2018;16(11):1665-75.

46. Chen B, Wei W, Huang X, Xie X, Kong Y, Dai D, et al. circEPSTI1 as a prognostic marker and mediator of triple-negative breast cancer progression. Theranostics. 2018;8(14):4003-15.

47. Zeng K, He B, Yang BB, Xu T, Chen X, Xu M, et al. The pro-metastasis effect of circANKS1B in breast cancer. Mol Cancer. 2018;17(1):160.

48. Xu J-Z, Shao C-C, Wang X-J, Zhao X, Chen J-Q, Ouyang Y-X, et al. circTADA2As suppress breast cancer progression and metastasis via targeting miR-203a-3p/SOCS3 axis. Cell Death Dis. 2019;10(3):175.

49. Zheng $X$, Huang $M$, Xing $L$, Yang $R$, Wang $X$, Jiang $R$, et al. The circRNA circSEPT9 mediated by E2F1 and EIF4A3 facilitates the carcinogenesis and development of triple-negative breast cancer. Mol Cancer. 2020;19(1):73.

50. Zheng SR, Huang QD, Zheng ZH, Zhang ZT, Guo GL. circGFRA1 affects the sensitivity of triple negative breast cancer cells to paclitaxel via the miR-361-5p/TLR4 pathway. J Biochem. 2021;mvaa148.https://doi. org/10.1093/jb/mvaa148.

51. Tang $H$, Huang $X$, Wang J, Yang L, Kong Y, Gao G, et al. circKIF4A acts as a prognostic factor and mediator to regulate the progression of triplenegative breast cancer. Mol Cancer. 2019;18(1):23.

52. Wang ST, Liu LB, Li XM, Wang YF, Xie PJ, Li Q, et al. Circ-ITCH regulates triple-negative breast cancer progression through the wnt/ $\beta$-catenin pathway. Neoplasma. 2019;66(2):232-9.

53. Wang S, Li Q, Wang Y, Li X, Wang R, Kang Y, et al. Upregulation of circUBAP2 predicts poor prognosis and promotes triple-negative breast cancer progression through the miR-661/MTA1 pathway. Biochem Biophys Res Commun. 2018;505(4):996-1002.

54. Kong Y, Yang L, Wei W, Lyu N, Zou Y, Gao G, et al. CircPLK1 sponges miR-296-5p to facilitate triple-negative breast cancer progression. Epigenomics. 2019;11(10):1163-76.

55. Wang Q, Li Z, Hu Y, Zheng W, Tang W, Zhai C, et al. Circ-TFCP2L1 promotes the proliferation and migration of triple negative breast cancer through sponging miR-7 by inhibiting PAK1. J Mammary Gland Biol Neoplasia. 2019;24(4):323-31.

56. Xiao W, Zheng S, Zou Y, Yang A, Xie X, Tang H, et al. CircAHNAK1 inhibits proliferation and metastasis of triple-negative breast cancer by modulating miR-421 and RASA1. Aging. 2019;11(24):12043-56.

57. Yang $R$, Xing $L$, Zheng $X$, Sun $Y$, Wang $X$, Chen J. The circRNA circAGFG1 acts as a sponge of miR-195-5p to promote triple-negative breast cancer progression through regulating CCNE1 expression. Mol Cancer. 2019;18(1):4.

58. Pei X, Wang X, Xue B, Zhang Y, Sun M, Li H. Circular RNA circ-ZEB1 acts as an oncogene in triple negative breast cancer via sponging miR-448. Int J Biochem Cell Biol. 2020;126:105798.

59. Liu P, Zou Y, Li X, Yang A, Ye F, Zhang J, et al. circGNB1 facilitates triplenegative breast cancer progression by regulating miR-141-5p-IGF1R axis. Front Genet. 2020;11:193.

60. Zhou Y, Liu X, Lan J, Wan Y, Zhu X. Circular RNA circRPPH1 promotes triple-negative breast cancer progression via the miR-556-5p/YAP1 axis. Am J Transl Res. 2020;12(10):6220-34.

61. Wang S, Liu F, Ma H, Cui X, Yang S, Qin R. circCDYL acts as a tumor suppressor in triple negative breast cancer by sponging miR-190a-3p and upregulating TP53INP1. Clin Breast Cancer. 2020;20(5):422-30.

62. Li X, Ren Z, Yao Y, Bao J, Yu Q. The circular RNA circEIF3M promotes breast cancer progression by promoting cyclin D1 expression. Aging. 2020;12(14):14775-90.

63. Dou D, Ren X, Han M, Xu X, Ge X, GuY, et al. CircUBE2D2 (hsa_ circ_0005728) promotes cell proliferation, metastasis and chemoresistance in triple-negative breast cancer by regulating miR-512-3p/CDCA3 axis. Cancer Cell Int. 2020;20:454. 
64. He D, Yang X, Kuang W, Huang G, Liu X, Zhang Y. The novel circular RNA Circ-PGAP3 promotes the proliferation and invasion of triple negative breast cancer by regulating the miR-330-3p/Myc axis. OncoTargets Therapy. 2020;13:10149-59.

65. Du WW, Zhang C, Yang W, Yong T, Awan FM, Yang BB. Identifying and characterizing circRNA-protein interaction. Theranostics. 2017;7(17):4183-91.

66. Du WW, Fang L, Yang W, Wu N, Awan FM, Yang Z, et al. Induction of tumor apoptosis through a circular RNA enhancing Foxo3 activity. Cell Death Differ. 2017;24(2):357-70.

67. Liu Y, Dong Y, Zhao L, Su L, Luo J. Circular RNA-MTO1 suppresses breast cancer cell viability and reverses monastrol resistance through regulating the TRAF4/Eg5 axis. Int J Oncol. 2018;53(4):1752-62.

68. Lei M, Zheng G, Ning Q, Zheng J, Dong D. Translation and functional roles of circular RNAs in human cancer. Mol Cancer. 2020;19(1):30.

69. Pamudurti NR, Bartok O, Jens M, Ashwal-Fluss R, Stottmeister C, Ruhe L, et al. Translation of CircRNAs. Mol Cell. 2017;66(1):9-21.e7.

70. Yang Y, Fan X, Mao M, Song X, Wu P, Zhang Y, et al. Extensive translation of circular RNAs driven by N(6)-methyladenosine. Cell Res. 2017;27(5):626-41.

71. Chen CY, Sarnow P. Initiation of protein synthesis by the eukaryotic translational apparatus on circular RNAs. Science (New York, NY). 1995;268(5209):415-7.

72. Abdelmohsen K, Panda AC, Munk R, Grammatikakis I, Dudekula DB, De $S$, et al. Identification of HuR target circular RNAs uncovers suppression of PABPN1 translation by CirCPABPN1. RNA Biol. 2017;14(3):361-9.

73. Legnini I, Di Timoteo G, Rossi F, Morlando M, Briganti F, Sthandier O, et al. Circ-ZNF609 is a circular RNA that can be translated and functions in myogenesis. Mol Cell. 2017;66(1):22-37.e9.

74. Ye F, Gao G, Zou Y, Zheng S, Zhang L, Ou X, et al. circFBXW7 inhibits malignant progression by sponging miR-197-3p and encoding a 185-aa protein in triple-negative breast cancer. Mol Therapy Nucleic Acids. 2019:18:88-98.

75. Li J, Ma M, Yang X, Zhang M, Luo J, Zhou H, et al. Circular HER2 RNA positive triple negative breast cancer is sensitive to Pertuzumab. Mol Cancer. 2020;19(1):142.

76. Zhang M, Zhao K, Xu X, Yang Y, Yan S, Wei P, et al. A peptide encoded by circular form of LINC-PINT suppresses oncogenic transcriptional elongation in glioblastoma. Nat Commun. 2018;9(1):4475.

77. Das A, Gorospe M, Panda AC. The coding potential of circRNAs. Aging. 2018;10(9):2228-9.

78. Zou Y, Zheng S, Xiao W, Xie X, Yang A, Gao G, et al. circRAD18 sponges miR-208a/3164 to promote triple-negative breast cancer progression through regulating IGF1 and FGF2 expression. Carcinogenesis. 2019;40(12):1469-79.

79. LiY, Shi P, Zheng T, Ying Z, Jiang D. Circular RNA hsa_circ_0131242 promotes triple-negative breast cancer progression by sponging hsamiR-2682. OncoTargets Therapy. 2020;13:4791-8.

80. Zheng X, Chen J. Expression of circRNA hsa_circ_0005320 in triplenegative breast cancer and its effect on cell proliferation. China Oncol. 2019;29(11):845-54

81. Zhang J, Xu HD, Xing XJ, Liang ZT, Xia ZH, Zhao Y. CircRNA_069718 promotes cell proliferation and invasion in triple-negative breast cancer by activating Wnt/beta-catenin pathway. Eur Rev Med Pharmacol Sci. 2019;23(12):5315-22.

82. Yang R, Chen J. Effects of circular RNA hsa_circ_0058514 on the development and progression of triple-negative breast cancer. China Oncol. 2019;29(1):9-18.

83. Lamouille S, Xu J, Derynck R. Molecular mechanisms of epithelial-mesenchymal transition. Nat Rev Mol Cell Biol. 2014;15(3):178-96.

84. Mittal V. Epithelial mesenchymal transition in tumor metastasis. Annu Rev Pathol. 2018:13:395-412.
85. Nusse $\mathrm{R}$, Clevers $\mathrm{H}$. Wnt/ $\beta$-catenin signaling, disease, and emerging therapeutic modalities. Cell. 2017;169(6):985-99.

86. Hwang HC, Clurman BE. Cyclin E in normal and neoplastic cell cycles. Oncogene. 2005;24(17):2776-86.

87. Gao D, Zhang X, Liu B, Meng D, Fang K, Guo Z, et al. Screening circular RNA related to chemotherapeutic resistance in breast cancer. Epigenomics. 2017;9(9):1175-88.

88. Rampurwala MM, Rocque GB, Burkard ME. Update on adjuvant chemotherapy for early breast cancer. Breast Cancer Basic Clin Res. 2014;8:125-33.

89. Zhang X, Su X, Guo Z, Jiang X, Li X. Circular RNA La-related RNA-binding protein 4 correlates with reduced tumor stage, as well as better prognosis, and promotes chemosensitivity to doxorubicin in breast cancer. J Clin Lab Anal. 2020;34(7):e23272.

90. Liang Y, Song X, Li Y, Su P, Han D, Ma T, et al. circKDM4C suppresses tumor progression and attenuates doxorubicin resistance by regulating miR-548p/PBLD axis in breast cancer. Oncogene. 2019;38(42):6850-66.

91. Gao D, Qi X, Zhang X, Fang K, Guo Z, Li L. hsa_circRNA_0006528 as a competing endogenous RNA promotes human breast cancer progression by sponging miR-7-5p and activating the MAPK/ERK signaling pathway. Mol Carcinog. 2019;58(4):554-64.

92. Li A, Yan Q, Zhao X, Zhong J, Yang H, Feng Z, et al. Decreased expression of PBLD correlates with poor prognosis and functions as a tumor suppressor in human hepatocellular carcinoma. Oncotarget. 2016;7(1):524-37.

93. Rajput S, Volk-Draper LD, Ran S. TLR4 is a novel determinant of the response to paclitaxel in breast cancer. Mol Cancer Ther. 2013;12(8):1676-87.

94. Luan T, Zhang X, Wang S, Song Y, Zhou S, Lin J, et al. Long non-coding RNA MIAT promotes breast cancer progression and functions as ceRNA to regulate DUSP7 expression by sponging miR-155-5p. Oncotarget. 2017:8(44):76153-64.

95. Yang W, Gong P, Yang Y, Yang C, Yang B, Ren L. Circ-ABCB10 contributes to paclitaxel resistance in breast cancer through let-7a-5p/DUSP7 axis. Cancer Manag Res. 2020;12:2327-37.

96. Ma J, Fang L, Yang Q, Hibberd S, Du WW, Wu N, et al. Posttranscriptional regulation of AKT by circular RNA angiomotin- like 1 mediates chemoresistance against paclitaxel in breast cancer cells. Aging. 2019;11(23):11369-81.

97. Yang W, Gu J, Wang X, Wang Y, Feng M, Zhou D, et al. Inhibition of circular RNA CDR1as increases chemosensitivity of 5-FU-resistant BC cells through up-regulating miR-7. J Cell Mol Med. 2019;23(5):3166-77.

98. Yu J, Shen W, Xu J, Gong B, Gao B, Zhu J. circUSP42 is downregulated in triple-negative breast cancer and associated with poor prognosis. Technol Cancer Res Treat. 2020;19:1533033820950827.

99. Hsiao KY, Sun HS, Tsai SJ. Circular RNA—new member of noncoding RNA with novel functions. Exp Biol Med. 2017;242(11):1136-41.

100. Wang J, Zhang Q, Zhou S, Xu H, Wang D, Feng J, et al. Circular RNA expression in exosomes derived from breast cancer cells and patients. Epigenomics. 2019;11(4):411-21.

101. Hu K, Liu X, Li Y, Li Q, Xu Y, Zeng W, et al. Exosomes mediated transfer of circ_UBE2D2 enhances the resistance of breast cancer to tamoxifen by binding to MiR-200a-3p. Med Sci Monit Int Med J Exp Clin Res. 2020;26:e922253.

102. Panda AC, De S, Grammatikakis I, Munk R, Yang X, Piao Y, et al. Highpurity circular RNA isolation method (RPAD) reveals vast collection of intronic circRNAs. Nucleic Acids Res. 2017:45(12):e116.

\section{Publisher's Note}

Springer Nature remains neutral with regard to jurisdictional claims in published maps and institutional affiliations. 